\title{
Catalytic Ester-Amide Exchange Using Group (IV) Metal Alkoxide-Activator Complexes
}

\author{
Chong Han, ${ }^{\dagger}$ Jonathan P. Lee, ${ }^{\dagger}$ Emil Lobkovsky, ${ }^{\ddagger}$ and John A. Porco, Jr. ${ }^{*}+$ \\ ${ }^{\dagger}$ Department of Chemistry and \\ Center for Chemical Methodology and Library Development, \\ Boston University, 590 Commonwealth Avenue, \\ Boston, Massachusetts 02215, \\ ${ }^{\ddagger}$ Department of Chemistry, Baker Laboratory, \\ Cornell University, Ithaca, New York 14583-1301 \\ Email:porco@chem.bu.edu
}

\section{Supporting Information}

\section{Contents:}

I. General Information. $\quad$ S-2

$\begin{array}{ll}\text { II. General procedure for ester-amide exchange. } & \mathrm{S}-2\end{array}$

III. Metal catalyst evaluation in the presence of HOBt. $\quad S-3$

IV. Compounds characterization. $\quad$ S-3

V. ${ }^{1} \mathrm{H}$ NMR studies. $\quad$ S-7

1. HOAt as additive. $\quad \mathrm{S}-7$

2. HOBt or HYP as additive. $\quad$ S-17

3. Reaction monitoring. $\quad$ S-18

VI. Variable temperature and 2D ${ }^{1} \mathrm{H}$ NMR studies. $\quad$ S-20

VII. X-ray crystal structure of complex $8 . \quad S-25$

VIII. Kinetic studies. $\quad$ S-29 
I. General Information: ${ }^{1} \mathrm{H}$ NMR spectra were recorded on a $400 \mathrm{MHz}$ spectrometer at ambient temperature. ${ }^{13} \mathrm{C}$ NMR spectra were recorded on a $75.0 \mathrm{MHz}$ spectrometer at ambient temperature with complete proton decoupling. Variable temperature, DQF-COSY, and T-ROESY ${ }^{1} \mathrm{H}$ NMR experiments were recorded on a $500 \mathrm{MHz}$ spectrometer. Chemical shifts for characterized compounds are reported in parts per million relative to $\mathrm{CDCl}_{3}\left({ }^{1} \mathrm{H}, \delta 7.27 ;{ }^{13} \mathrm{C}, \delta 77.23\right)$ unless otherwise stated. Data for ${ }^{1} \mathrm{H}$ NMR are reported as follows: chemical shift, integration, multiplicity $($ app $=$ apparent, $\mathrm{br}=$ broad, ovrlp $=$ overlapping, $\mathrm{s}=$ singlet, $\mathrm{d}=$ doublet, $\mathrm{t}=$ triplet, $\mathrm{q}=$ quartet, $\mathrm{m}=$ multiplet) and coupling constants. Infrared spectra were recorded on a Nicolet Nexus 670 FT-IR spectrophotometer. React-IR spectra were recorded using a METTLER TOLEDO Autochem React-IR 3.0 spectrophotometer. Enantiomeric excess values were determined by chiral HPLC analysis performed on an Agilent 1100 series HPLC (column: Chiralcel ${ }^{\circledR}$ OD, Chiral technologies Inc., $24 \mathrm{~cm}$ x $4.6 \mathrm{~mm}$ ). High-resolution mass spectra were obtained in the Boston University Mass Spectrometry Laboratory using a Finnegan MAT-90 spectrometer. Analytical thin layer chromatography was performed on $0.25 \mathrm{~mm}$ silica gel $60-\mathrm{F}$ plates. Flash chromatography was performed using 200 400 mesh silica gel (Scientific Absorbents Incorporated). $\mathrm{Zr}(\mathrm{Ot} \text {-Bu})_{4}$ was obtained from Strem Chemicals and 1-hydroxy-7-azabenzotriazole (HOAt) was obtained from TCI-America Chemicals. Benzene- $d_{6}$ was distilled from calcium hydride. Yields refer to chromatographically and spectroscopically pure materials unless otherwise stated. All other reagents were used as supplied by Sigma-Aldrich, Alfa-Aesar, Lancaster, Fluka, Strem, and Bachem Chemicals. All reactions were performed in oven-dried glassware under an argon atmosphere unless otherwise noted. Compounds $\mathbf{1} \mathbf{b}^{1}, \mathbf{1 c}^{2}$, and $\mathbf{1} \mathbf{d}^{3}$ were prepared according to procedures.

\section{General procedure for ester-amide exchange catalyzed by $\mathrm{Zr}(\mathrm{Ot} \text {-Bu })_{4}$ and $\mathrm{HOAt}$ as additive (Table 3):}

To a solution of carboxylic ester $(1.00 \mathrm{mmol})$ and amine $(1.10 \mathrm{mmol})$ in $1 \mathrm{~mL}(1.00 \mathrm{M}$ in ester substrate $)$ of toluene was added HOAt $(0.05 \mathrm{mmol})$ followed by $\mathrm{Zr}(\mathrm{Ot}-\mathrm{Bu})_{4}(0.05 \mathrm{mmol})$. The reaction was stirred at the desired temperature for the indicated time and quenched by addition of $\mathrm{MeOH}(2 \mathrm{~mL})$ and $\mathrm{CH}_{2} \mathrm{Cl}_{2}(2 \mathrm{~mL})$. The reaction mixture was filtered through a silica gel pad and concentrated in vacuo. Conversions were determined by ${ }^{1} \mathrm{H}$ NMR analysis of the crude reaction mixtures. Amide products were isolated by flash column chromatography using silica gel.

(1) Shieh, W-C.; Dell, S.; Repič, O. J. Org. Chem. 2002, 67, 2188.

(2) Maguire, A. R.; Plunkett, S. J.; Papot, S.; Clynes, M.; O’Coonor, R.; Touhey, S. Bioorg. Med. Chem. 2001, 9, 745.

(3) Kim, H. K.; Na, H. S.; Park, M. S.; Oh, T. K.; Lee, T. S. J. Mol. Catal. B: Enzym. 2004, 27, 237. 


\section{Metal catalyst evaluation in the presence of HOBt:}

$\underset{1 \mathbf{1 a}}{\mathrm{O}} \frac{\begin{array}{l}10 \text { mol\% catalyst } \\ \text { toluene }(0.5 \mathrm{M}), 60^{\circ} \mathrm{C}, 1 \mathrm{~h}\end{array}}{\mathrm{Ph}}$

\begin{tabular}{|l|c|l|c|}
\hline \multicolumn{1}{|c|}{ catalyst } & ${\text { conversion }(\%)^{\mathrm{a}}}$ & \multicolumn{1}{c|}{ catalyst } & ${\text { conversion }(\%)^{a}}^{a}$ \\
\hline $\mathrm{Zr}(\mathrm{Ot}-\mathrm{Bu})_{4}+2 \mathrm{HOBt}$ & 79 & $\mathrm{HfCl}_{4}+2 \mathrm{HOBt}$ & 33 \\
\hline $\mathrm{Zr}(\mathrm{Oi}-\mathrm{Pr})_{4}+2 \mathrm{HOBt}$ & 65 & $\mathrm{Sb}(\mathrm{Oi}-\mathrm{Pr})_{3}+2 \mathrm{HOBt}$ & 21 \\
\hline $\mathrm{Hf}(\mathrm{Ot}-\mathrm{Bu})_{4}+2 \mathrm{HOBt}$ & 80 & $\mathrm{ZnEt}_{2}+2 \mathrm{HOBt}$ & 9 \\
\hline $\mathrm{Hf}(\mathrm{Oi}-\mathrm{Pr})_{4}+2 \mathrm{HOBt}$ & 60 & $\mathrm{Cu}(\mathrm{OBt})_{2}$ & 26 \\
\hline
\end{tabular}

${ }^{a}$ Conversion based on ${ }^{1} \mathrm{H}$ NMR analysis of the crude reaction mixture.

\section{Compound characterization:}

Compounds $\mathbf{2 a},{ }^{4} \mathbf{2} \mathbf{i},{ }^{5} \mathbf{2} \mathbf{l},{ }^{6} \mathbf{2} \mathbf{m},{ }^{7} \mathbf{2 0},{ }^{8}$ and $\mathbf{2} \mathbf{p},{ }^{9}$ were characterized by comparing their ${ }^{1} \mathrm{H}$ NMR and ${ }^{13} \mathrm{C}$ NMR data with those previously reported in the literature. Compound $\mathbf{2 q}$ were characterized by comparing its ${ }^{1} \mathrm{H}$ NMR and ${ }^{13} \mathrm{C}$ NMR data with spectra obtained from a commercially available sample (Aldrich).

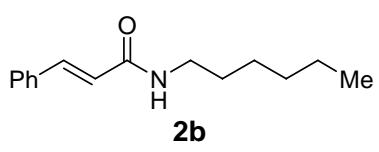

Purified by flash chromatography on silica gel (hexane: EtOAc= $3: 1$ ). mp. $38-39^{\circ} \mathrm{C}$; IR (neat): 3279, 3082, 2929, 2858, 1656, 1619, 1557, 1451, 1341, 1224, 979, 865, 762, $682 \mathrm{~cm}^{-1} ;{ }^{1} \mathrm{H}$ NMR: $\delta 7.61(1 \mathrm{H}, \mathrm{d}, J=15.2 \mathrm{~Hz})$, 7.47-7.45 (2H, m), 7.35-7.30 (3H, m), $6.38(1 \mathrm{H}, \mathrm{d}, J=15.2 \mathrm{~Hz}), 5.69(1 \mathrm{H}$, br), $3.37(2 \mathrm{H}, \mathrm{dt}, J=7.2,6.0 \mathrm{~Hz}), 1.57(2 \mathrm{H}, \mathrm{tt}, J=7.2,7.2 \mathrm{~Hz}), 1.36-1.25(6 \mathrm{H}, \mathrm{m}), 0.87(3 \mathrm{H}, \mathrm{t}, J=6.8$ Hz) ppm; ${ }^{13} \mathrm{C}$ NMR: $\delta$ 166.2, 140.4, 134.9, 129.5, 128.7 (2C), 127.7 (2C), 121.2, 39.9, 31.6, 29.7,

(4) Matsuura, I.; Ueda, T.; Murakami, N.; Nagai, S.; Sakakibara, J. J. Chem. Soc. Perkin Trans. 1 1991, 2821.

(5) Prashad, M.; Hu, B.; Har, D.; Repič, O.; Blacklock, T. J. Tetrahedron Lett. 2000, 41, 9957.

(6) Ishihara, K.; Ohara, S.; Yamamoto, H. J. Org. Chem. 1996, 61, 4196.

(7) Kim, J. M.; Wilson, T. E.; Norman, T. C.; Schultz, P. G. Tetrahedron Lett. 1996, 37, 5309.

(8) Sanchez, R.; Vest, G.; Despres, L. Synth. Commun. 1989, 19, 2909.

(9) Kunishima, M.; Kawachi, C.; Hioki, K.; Terao, K.; Tani, S. Tetrahedron 2001, 57, 1551. 
26.8, 22.6, 14.0 ppm; CI-HRMS calcd. for $\mathrm{C}_{15} \mathrm{H}_{22} \mathrm{NO}[\mathrm{M}+\mathrm{H}]^{+}:$232.1701, found 232.1707.

Purified by flash chromatography on silica gel (hexane: EtOAc=1:2). mp.

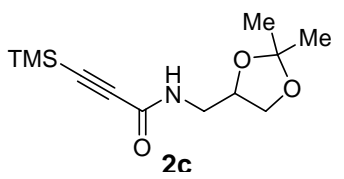

66-68 ${ }^{\circ} \mathrm{C}$; IR (neat): 3285, 2986, 2961, 2937, 1636, 1539, 1380, 1372, 1252, 1217, 1158, 1074, 847, $762 \mathrm{~cm}^{-1} ;{ }^{1} \mathrm{H}$ NMR: $\delta 6.14(1 \mathrm{H}, \mathrm{bs}), 4.20(1 \mathrm{H}, \mathrm{tt}, J$ $=7.2,6.8 \mathrm{~Hz}), 4.01(1 \mathrm{H}, \mathrm{dd}, J=7.6,7.2 \mathrm{~Hz}), 3.60(1 \mathrm{H}, \mathrm{dd}, J=7.6,7.2 \mathrm{~Hz})$, $3.60(1 \mathrm{H}$, dd, ovrlp, $J=14.0,6.8 \mathrm{~Hz}), 3.22(1 \mathrm{H}, \mathrm{dd}, J=14.0,6.8 \mathrm{~Hz}), 1.41$ $(3 \mathrm{H}, \mathrm{s}), 1.31(3 \mathrm{H}, \mathrm{s}), 0.18(9 \mathrm{H}, \mathrm{s}) \mathrm{ppm} ;{ }^{13} \mathrm{C} \mathrm{NMR}: \delta 153.2,109.8,97.5,92.2,66.9,42.3,27.0,25.4$, -0.52 ppm; CI-HRMS calcd. for $\mathrm{C}_{12} \mathrm{H}_{22} \mathrm{NO}_{3} \mathrm{Si}[\mathrm{M}+\mathrm{H}]^{+}: 256.1369$, found 256.1369.

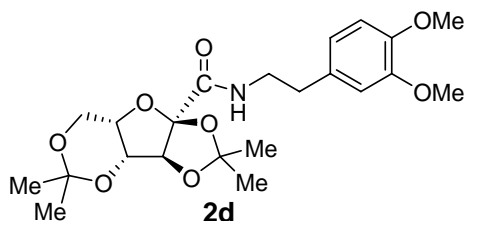

Purified by flash chromatography on silica gel (hexane: EtOAc= 3:2). IR (neat): 3413, 2992, 2938, 1692, 1516, 1454, 1375, 1260, $1237,1185,1145,1121,1094,1029,915,868,831,763 \mathrm{~cm}^{-1} ;{ }^{1} \mathrm{H}$ NMR: $\delta 7.20(1 \mathrm{H}, \mathrm{bs}), 6.75-6.71(3 \mathrm{H}, \mathrm{m}), 4.55(1 \mathrm{H}, \mathrm{s}), 4.27(1 \mathrm{H}, \mathrm{d}$, $J=1.6 \mathrm{~Hz}), 4.13(1 \mathrm{H}, \mathrm{d}, J=1.6 \mathrm{~Hz}), 4.06(1 \mathrm{H}, \mathrm{dd}, J=14.0,1.6 \mathrm{~Hz})$, $4.00(1 \mathrm{H}, \mathrm{d}, J=14.0 \mathrm{~Hz}), 3.83(3 \mathrm{H}, \mathrm{s}), 3.81(3 \mathrm{H}, \mathrm{s}), 3.57(1 \mathrm{H}, \mathrm{ddt}, J=13.2,7.6,6.0 \mathrm{~Hz}), 3.37(1 \mathrm{H}$, ddt, $J=13.2,7.6,6.0 \mathrm{~Hz}), 2.74(2 \mathrm{H}, \mathrm{dd}, J=7.6,7.6 \mathrm{~Hz}), 1.52(3 \mathrm{H}, \mathrm{s}), 1.50(3 \mathrm{H}, \mathrm{s}), 1.38(3 \mathrm{H}, \mathrm{s}), 1.21$ $(3 \mathrm{H}, \mathrm{s})$ ppm; ${ }^{13} \mathrm{C}$ NMR: $\delta 167.5,149.2,147.8,131.4,120.9,114.7,112.1,111.6,110.8,97.7,88.1$, 73.7, 72.6, 60.3, 56.1, 56.0, 44.5, 35.5, 29.3, 27.3, 25.9, 18.7 ppm; CI-HRMS calcd. for $\mathrm{C}_{22} \mathrm{H}_{31} \mathrm{NO}_{8}$ $[\mathrm{M}+\mathrm{H}]^{+}: 438.2128$, found 438.2145. $[\alpha]_{\mathrm{D}}^{25}=-12.8\left(c=1.88, \mathrm{CHCl}_{3}\right)$

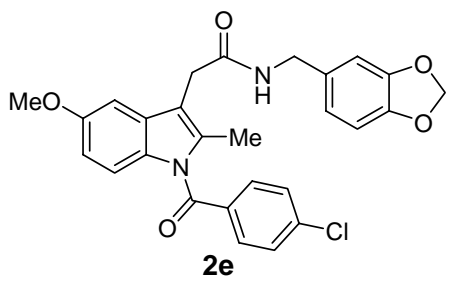

Purified by flash chromatography on silica gel (hexane: EtOAc $=5: 1$ ). mp. $132-134{ }^{\circ} \mathrm{C}$; IR (neat): 3324, 2949, 1733, 1644, 1595, 1539, 1502, $1487,1443,1306,1251,1217,1170,1095,1037,1015,926,847$ $\mathrm{cm}^{-1}$; ${ }^{1} \mathrm{H}$ NMR: $\delta 8.03(1 \mathrm{H}, \mathrm{bs}), 7.66(2 \mathrm{H}, \mathrm{d}, J=8.4 \mathrm{~Hz}), 7.31(2 \mathrm{H}, \mathrm{d}, J$ $=8.4 \mathrm{~Hz}), 7.06(1 \mathrm{H}, \mathrm{d}, J=8.8 \mathrm{~Hz}), 6.94(1 \mathrm{H}, \mathrm{d}, J=2.0 \mathrm{~Hz}), 6.76(1 \mathrm{H}$, s), 6.73-6.70 (3H, m), $5.89(2 \mathrm{H}, \mathrm{s}), 4.44(2 \mathrm{H}, \mathrm{d}, J=6.0 \mathrm{~Hz}), 3.81(3 \mathrm{H}$, s), $3.62(2 \mathrm{H}, \mathrm{s}), 2.31(3 \mathrm{H}, \mathrm{s}) \mathrm{ppm} ;{ }^{13} \mathrm{C} \mathrm{NMR}: \delta 172.9,166.6,154.3$, 148.2, 147.3, 138.0, 133.9, 132.9, 132.1, 130.4, 129.0 (2C), 128.7 (2C), 121.4, 111.3, 111.0, 108.7, 108.6, 104.4, 101.3, 100.6, 56.1, 52.1, 44.2, 30.5, 12.0 ppm; CI-HRMS calcd. for $\mathrm{C}_{27} \mathrm{H}_{24} \mathrm{ClN}_{2} \mathrm{O}_{2}$ $[\mathrm{M}+\mathrm{H}]^{+}:$491.1373, found 491.1326.

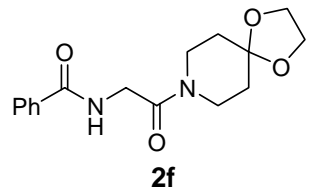

Purified by flash chromatography on silica gel $\left(\mathrm{CH}_{2} \mathrm{Cl}_{2}\right.$ : acetone $=6: 1,1 \%$ $\mathrm{Et}_{3} \mathrm{~N}$ ). mp. 88-90 ${ }^{\circ} \mathrm{C}$; IR (neat): $3339,2962,2932,2884,1642,1579,1540$, $1475,1443,1362,1339,1309,1268,1230,1146,1105,1033,945,754,713$ $\mathrm{cm}^{-1}$; ${ }^{1} \mathrm{H}$ NMR: $\delta 7.76(2 \mathrm{H}, \mathrm{d}, J=7.2 \mathrm{~Hz}), 7.41(1 \mathrm{H}, \mathrm{dd}, J=7.2,7.2 \mathrm{~Hz}), 7.34$ 
$(2 \mathrm{H}, \mathrm{dd}, J=7.2,7.2 \mathrm{~Hz}), 7.34(1 \mathrm{H}, \mathrm{bs}, \mathrm{ovrlp}), 4.17(2 \mathrm{H}, \mathrm{d}, J=4.0 \mathrm{~Hz}), 3.89(4 \mathrm{H}, \mathrm{s}), 3.65(2 \mathrm{H}, \mathrm{t}, J=6.0$ $\mathrm{Hz}), 3.43(2 \mathrm{H}, \mathrm{t}, J=6.0 \mathrm{~Hz}), 1.63(4 \mathrm{H}, \mathrm{t}, J=6.0 \mathrm{~Hz}) \mathrm{ppm} ;{ }^{13} \mathrm{C}$ NMR: $\delta 167.4,166.5,134.1,131.8$, 128.7 (2C), 127.3 (2C), 106.8, 64.7 (2C), 42.6, 41.9, 40.5, 35.4, 34.9 ppm; CI-HRMS calcd. for $\mathrm{C}_{16} \mathrm{H}_{21} \mathrm{~N}_{2} \mathrm{O}_{4}[\mathrm{M}+\mathrm{H}]^{+}:$305.1501, found 305.1504 .

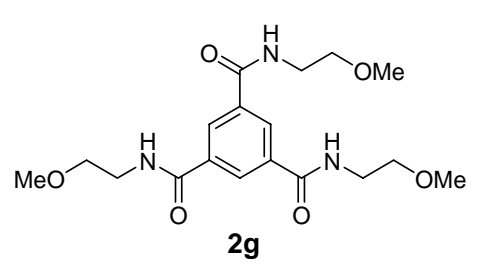

Purified by flash chromatography on silica gel $\left(\mathrm{CH}_{2} \mathrm{Cl}_{2}: \mathrm{MeOH}=\right.$ 20:1). mp. 147-148 ${ }^{\circ} \mathrm{C}$; IR (neat): 3251, 3074, 2931, 2882, 1636, $1559,1457,1297,1199,1127,1987,1022,922,718,683 \mathrm{~cm}^{-1} ;{ }^{1} \mathrm{H}$ NMR: $\delta 8.02(3 \mathrm{H}, \mathrm{s}), 7.57(3 \mathrm{H}, \mathrm{bs}), 3.59(6 \mathrm{H}, \mathrm{t}, J=4.4 \mathrm{~Hz}), 3.52$ $(6 \mathrm{H}, \mathrm{t}, J=4.4 \mathrm{~Hz}), 3.36(9 \mathrm{H}, \mathrm{s}) \mathrm{ppm} ;{ }^{13} \mathrm{C}$ NMR: $\delta 165.7$ (3C), 134.9 (3C), 127.9 (3C), 71.2 (3C), 58.7 (3C), 40.2 (3C) ppm; CI-HRMS calcd. for $\mathrm{C}_{18} \mathrm{H}_{28} \mathrm{~N}_{3} \mathrm{O}_{6}[\mathrm{M}+\mathrm{H}]^{+}: 382.1978$, found 382.1999.

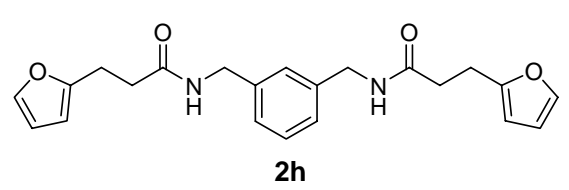

Purified by flash chromatography on silica gel $\left(\mathrm{CH}_{2} \mathrm{Cl}_{2}\right.$ : acetone $=8: 1) . \mathrm{mp} .135-136^{\circ} \mathrm{C}$; IR (neat): $3291,3078,2927$, 1642, 1551, 1444, 1223, 1151, 1015, 924, 731, $701 \mathrm{~cm}^{-1} ;{ }^{1} \mathrm{H}$ NMR: $\delta 7.21(2 \mathrm{H}, \mathrm{d}, J=2.4 \mathrm{~Hz}), 7.16(1 \mathrm{H}, \mathrm{dd}, J=7.6,7.6$

$\mathrm{Hz}), 7.00(2 \mathrm{H}, \mathrm{d}, J=7.6 \mathrm{~Hz}), 6.92(1 \mathrm{H}, \mathrm{s}), 6.34(2 \mathrm{H}, \mathrm{bs}), 6.21(2 \mathrm{H}, \mathrm{dd}, J=2.4 \mathrm{~Hz}), 5.95(2 \mathrm{H}, \mathrm{d}, J=2.4$ $\mathrm{Hz}), 4.21(4 \mathrm{H}, \mathrm{d}, J=5.6 \mathrm{~Hz}), 2.91(4 \mathrm{H}, \mathrm{t}, J=3.6 \mathrm{~Hz}), 2.47(4 \mathrm{H}, \mathrm{t}, J=3.6 \mathrm{~Hz}) \mathrm{ppm} ;{ }^{13} \mathrm{C} \mathrm{NMR}: \delta 172.1$ (2C), 154.6 (2C), 141.4 (2C), 138.9 (2C), 129.2, 127.1, 126.9 (2C), 110.5 (2C), 105.7 (2C), 43.5 (2C), 35.0 (2C), 24.2 (2C) ppm; CI-HRMS calcd. for $\mathrm{C}_{22} \mathrm{H}_{25} \mathrm{~N}_{2} \mathrm{O}_{4}[\mathrm{M}+\mathrm{H}]^{+}:$: 381.1814, found 381.1812.

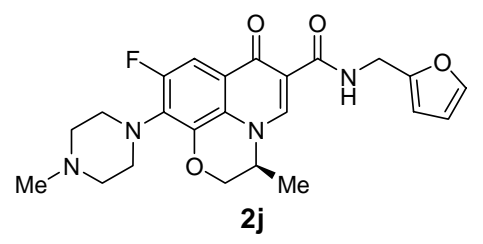

Purified by flash chromatography on silica gel (EtOAc: $\mathrm{CH}_{2} \mathrm{Cl}_{2}$ : $\left.\mathrm{MeOH}: \mathrm{Et}_{3} \mathrm{~N}=5: 2: 2: 1\right) . \mathrm{mp} .155-15{ }^{\circ} \mathrm{C}$; IR (neat): 3229, 2935 , $2843,2795,1655,1618,1604,1541,1474,1449,1373,1348,1294$, 1238, 1149, 1048, $1007 \mathrm{~cm}^{-1}$; ${ }^{1} \mathrm{H}$ NMR: $\delta 10.29(1 \mathrm{H}, \mathrm{t}, J=6.0 \mathrm{~Hz})$, $8.61(1 \mathrm{H}, \mathrm{s}), 7.67(1 \mathrm{H}, \mathrm{d}, J=12.4 \mathrm{~Hz}), 7.33(1 \mathrm{H}, \mathrm{d}, J=2.0 \mathrm{~Hz}), 6.27$ $(1 \mathrm{H}, \mathrm{d}, J=2.0 \mathrm{~Hz}), 6.24(1 \mathrm{H}, \mathrm{d}, J=2.0 \mathrm{~Hz}), 4.64(1 \mathrm{H}, \mathrm{dd}, J=6.0,16.0 \mathrm{~Hz}), 4.59(1 \mathrm{H}, \mathrm{dd}, J=6.0,16.0$ $\mathrm{Hz}$, 4.39-4.26 (3H, m), 3.39 (4H, bs), $2.62(4 \mathrm{H}, \mathrm{bs}), 2.40(3 \mathrm{H}, \mathrm{s}), 1.55(3 \mathrm{H}, \mathrm{d}, J=6.8 \mathrm{~Hz}) \mathrm{ppm} ;{ }^{13} \mathrm{C}$ NMR: $\delta$ 175.3, 165.1, 157.6, 154.3, 152.2, 144.3, 142.1, 139.7, 132.2, 68.3, 55.9 (2C), 55.0, 50.8 (2C), 46.6, 36.4, 18.5 ppm; CI-HRMS calcd. for $\mathrm{C}_{23} \mathrm{H}_{26} \mathrm{FN}_{4} \mathrm{O}_{4}: 440.1860$, found 440.1885. $[\alpha]_{\mathrm{D}}{ }^{25}=-64.7$ $\left(c=0.53, \mathrm{CHCl}_{3}\right)$

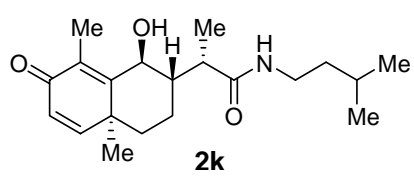

Purified by flash chromatography on silica gel $\left(\mathrm{CH}_{2} \mathrm{Cl}_{2}\right.$ : acetone $\left.=6: 1\right)$. mp. 178-179 ${ }^{\circ} \mathrm{C}$; IR (neat): 3335, 2955, 2870, 1660, 1621, 1540, 1457, 1373, 1306, 1165, 1136, $834 \mathrm{~cm}^{-1}$; ${ }^{1} \mathrm{H}$ NMR: $\delta 6.62(1 \mathrm{H}, \mathrm{d}, J=9.6 \mathrm{~Hz})$, $6.17(1 \mathrm{H}, \mathrm{d}, J=9.6 \mathrm{~Hz}), 5.77(1 \mathrm{H}, \mathrm{bs}), 4.48(1 \mathrm{H}, \mathrm{d}, J=9.6 \mathrm{~Hz}), 3.64$ 
$(1 \mathrm{H}, \mathrm{bs}), 3.27(1 \mathrm{H}, \mathrm{ddt}, J=13.2,7.2,5.6 \mathrm{~Hz}), 3.24(1 \mathrm{H}, \mathrm{ddt}, J=13.2,7.2,5.6 \mathrm{~Hz}), 2.57(1 \mathrm{H}, \mathrm{dq}, J=$ $7.2,4.0 \mathrm{~Hz}), 1.94-1.86(1 \mathrm{H}, \mathrm{m}), 1.74-1.53(5 \mathrm{H}, \mathrm{m}), 1.36(2 \mathrm{H}, \mathrm{dt}, J=7.2,7.2 \mathrm{~Hz}), 1.22(3 \mathrm{H}, \mathrm{d}, J=7.2$ $\mathrm{Hz}), 1.18(3 \mathrm{H}, \mathrm{s}), 0.87(6 \mathrm{H}, \mathrm{d}, J=6.8 \mathrm{~Hz}) \mathrm{ppm} ;{ }^{13} \mathrm{C}$ NMR: $\delta 188.0,176.4,159.5,156.7,129.8,125.8$, 74.5, 50.2, 42.8, 42.2, 38.7, 38.3, 38.2, 26.1, 23.7, 23.5, 22.7, 22.6, 14.4, 11.6 ppm; CI-HRMS calcd. for $\mathrm{C}_{20} \mathrm{H}_{32} \mathrm{NO}_{3}[\mathrm{M}+\mathrm{H}]^{+}: 334.2382$, found 334.2359. $[\alpha]_{\mathrm{D}}{ }^{25}=-48.3\left(c=1.12, \mathrm{CHCl}_{3}\right)$

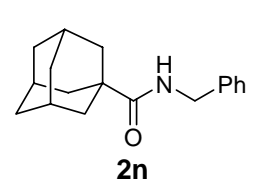

Purified by flash chromatography on silica gel (hexane: EtOAc $=3: 1$ ). mp. 172-173 ${ }^{\circ} \mathrm{C}$; IR (neat): 3341, 2903, 2848, 1632, 1531, 1495, 1451, 1412, 1283, 1238, 1104, 1002, 716, 692, $667 \mathrm{~cm}^{-1}$; ${ }^{1} \mathrm{H}$ NMR: $\delta$ 7.29-7.19 $(5 \mathrm{H}, \mathrm{m}), 5.99(1 \mathrm{H}$, bs), 4.38 (2H, d, $J=4.8 \mathrm{~Hz}), 1.99$ (3H, bs), 1.84 (6H, bs), 1.67 (6H, dd, $J=12.0$,

$11.2 \mathrm{~Hz}$ ) ppm; ${ }^{13} \mathrm{C}$ NMR: $\delta$ 178.1, 139.0, 128.9 (2C), 127.8 (2C), 127.6, 43.5, 40.9, 39.5 (3C), 36.8 (3C), 28.4 (3C) ppm; CI-HRMS calcd. for $\mathrm{C}_{18} \mathrm{H}_{24} \mathrm{NO}[\mathrm{M}]^{+}: 270.1780$, found 270.1761 . 


\section{V. ${ }^{1}$ H NMR studies:}

1. HOAt as additive.

a)

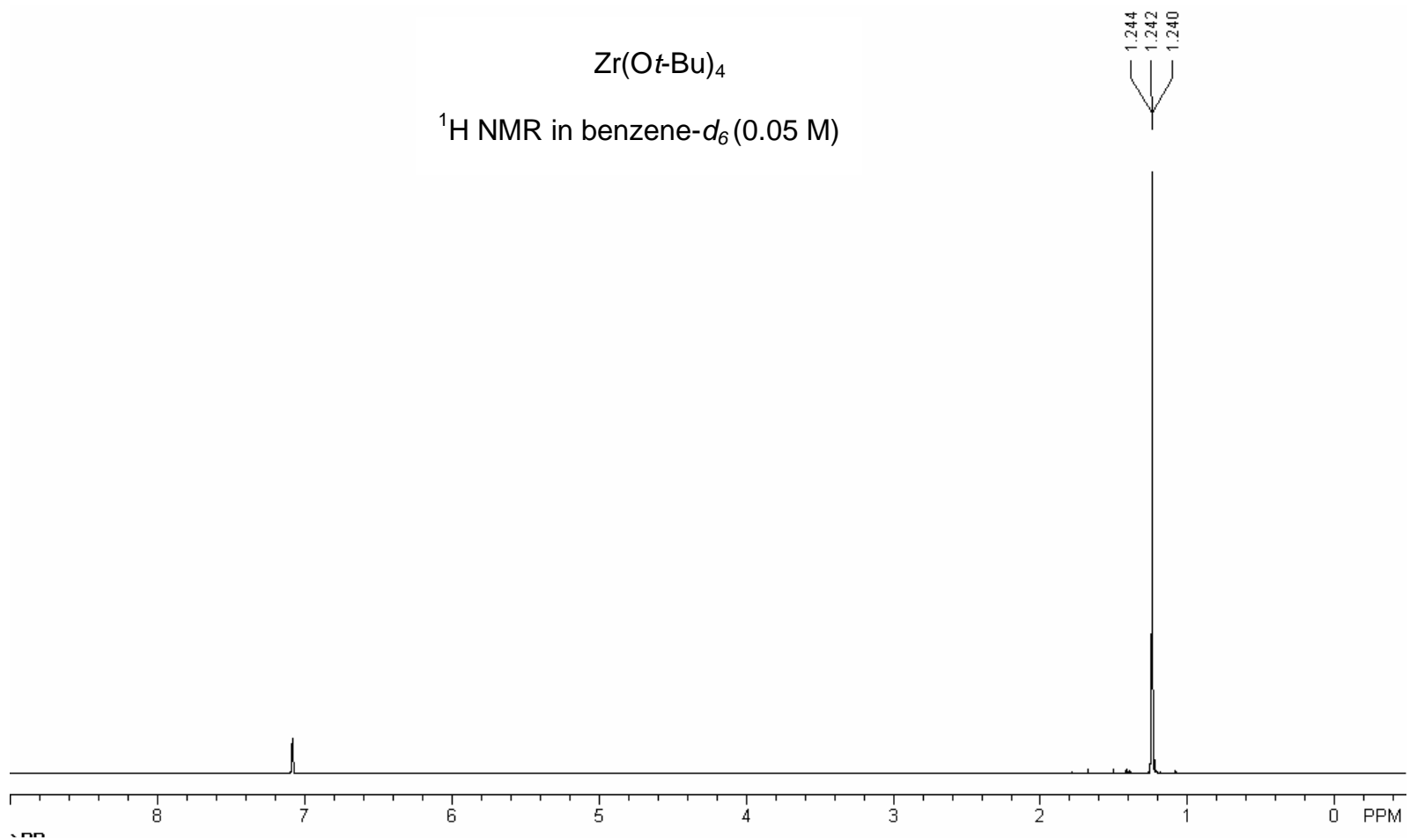

b)

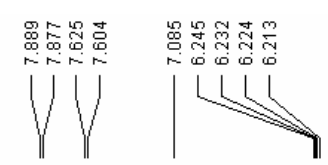

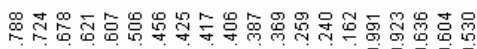

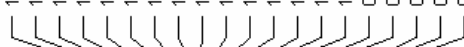

iii ilit

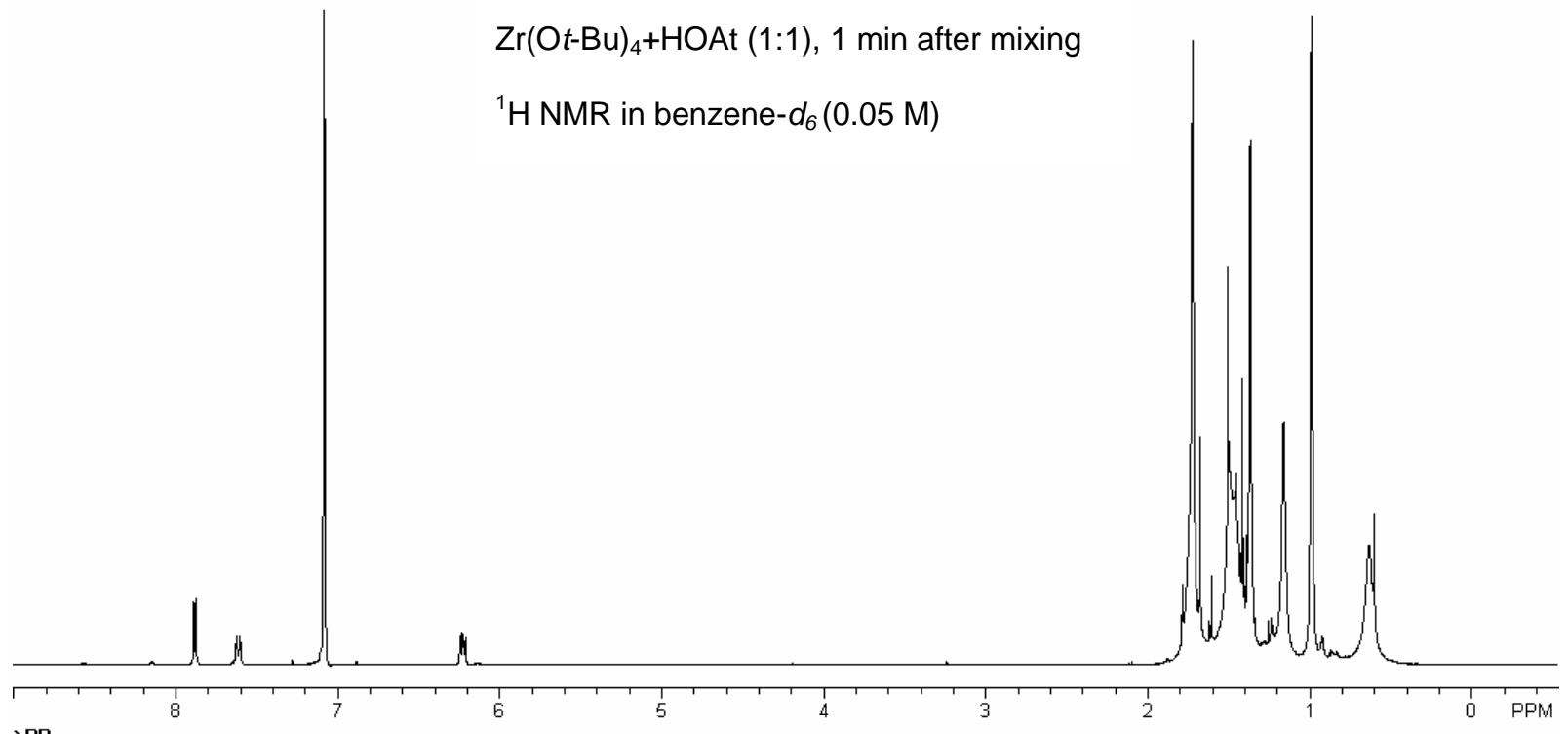


c)
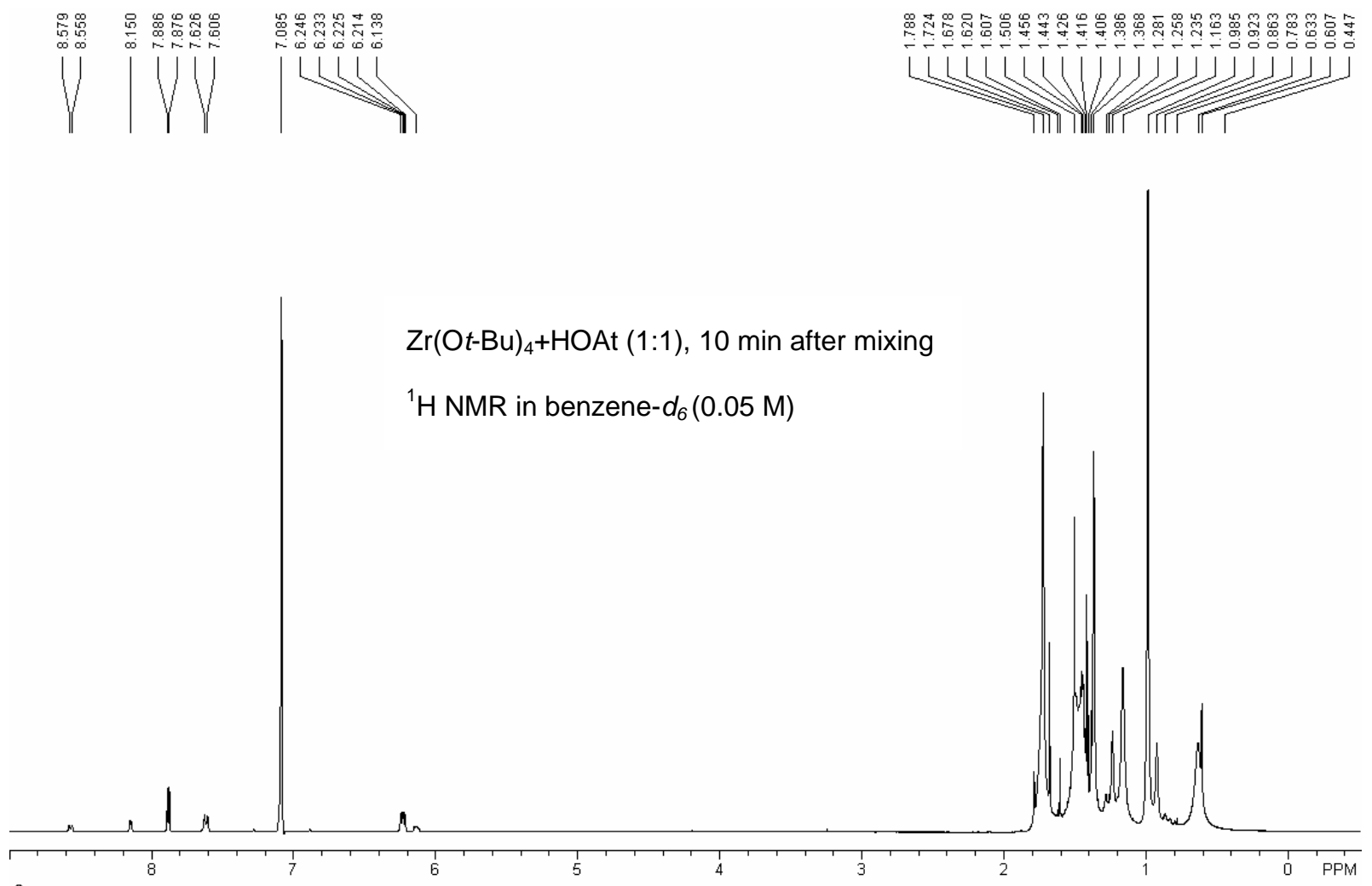

d)

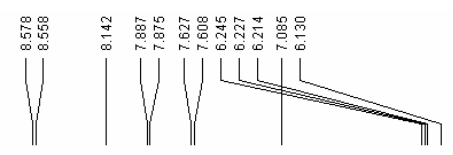

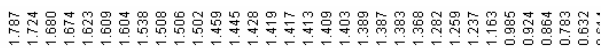

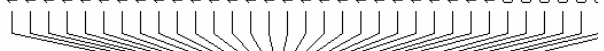

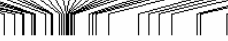

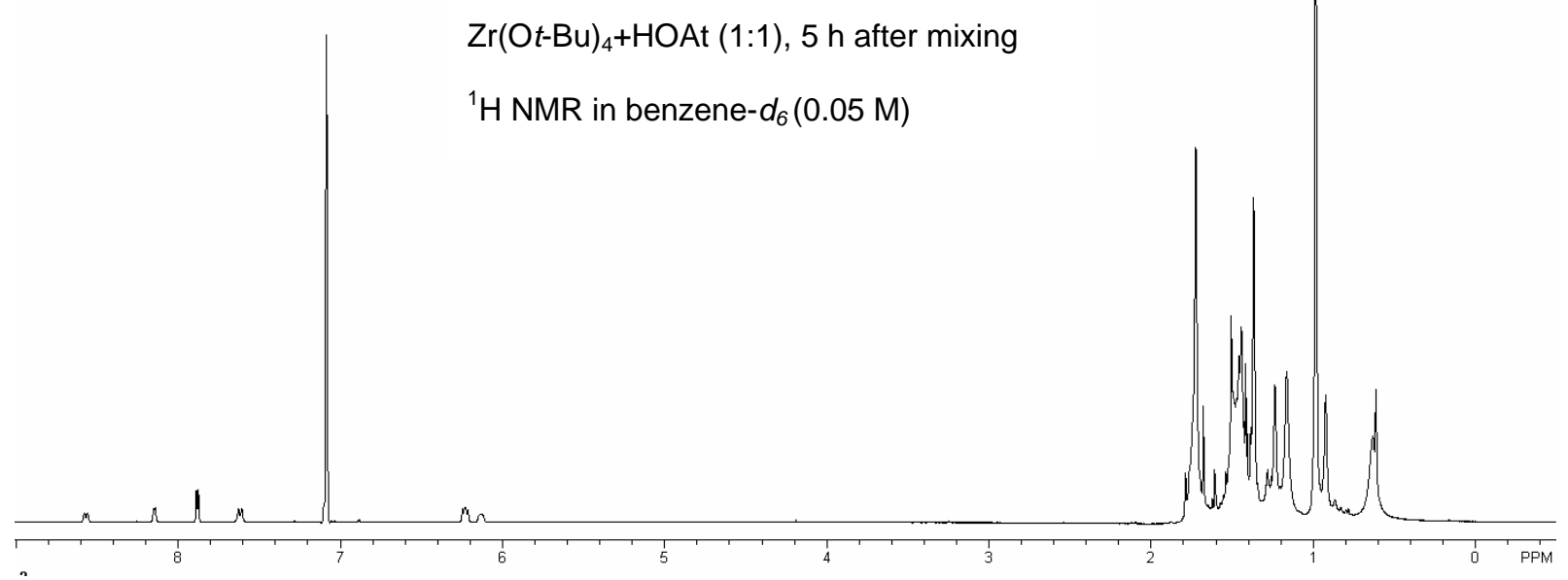


e)

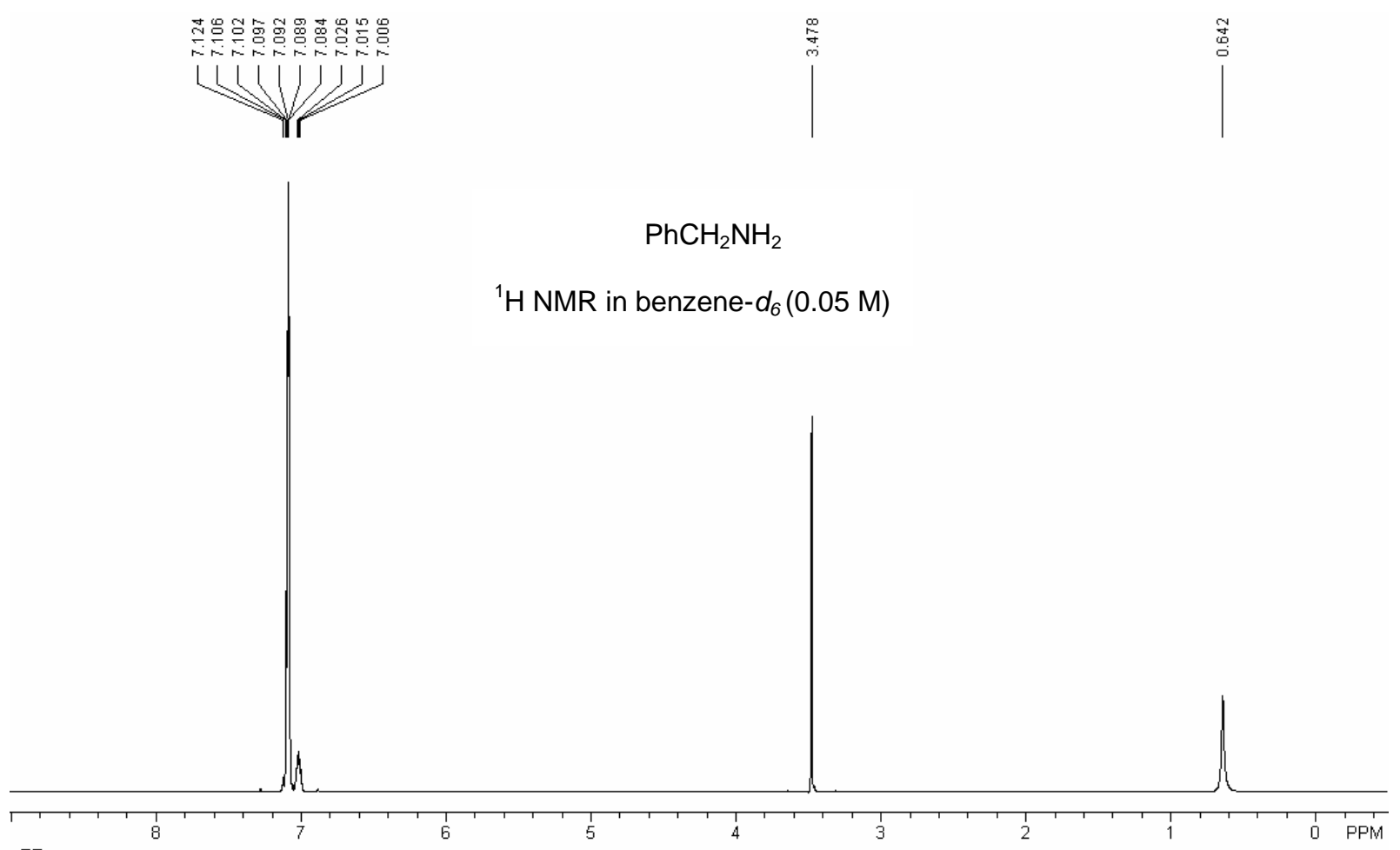

f)
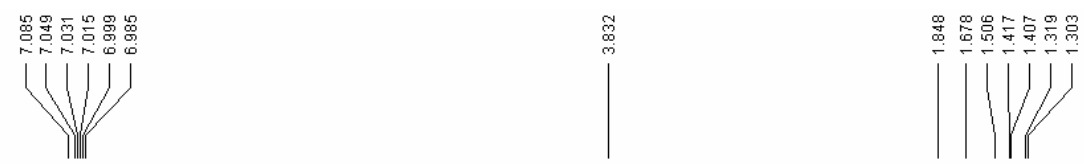

$\mathrm{Zr}(\mathrm{Ot}-\mathrm{Bu})_{4}+\mathrm{PhCH}_{2} \mathrm{NH}_{2}(1: 1)$

${ }^{1} \mathrm{H}$ NMR in benzene- $d_{6}(0.05 \mathrm{M})$

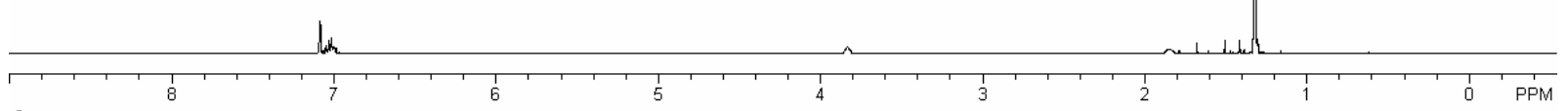


g)

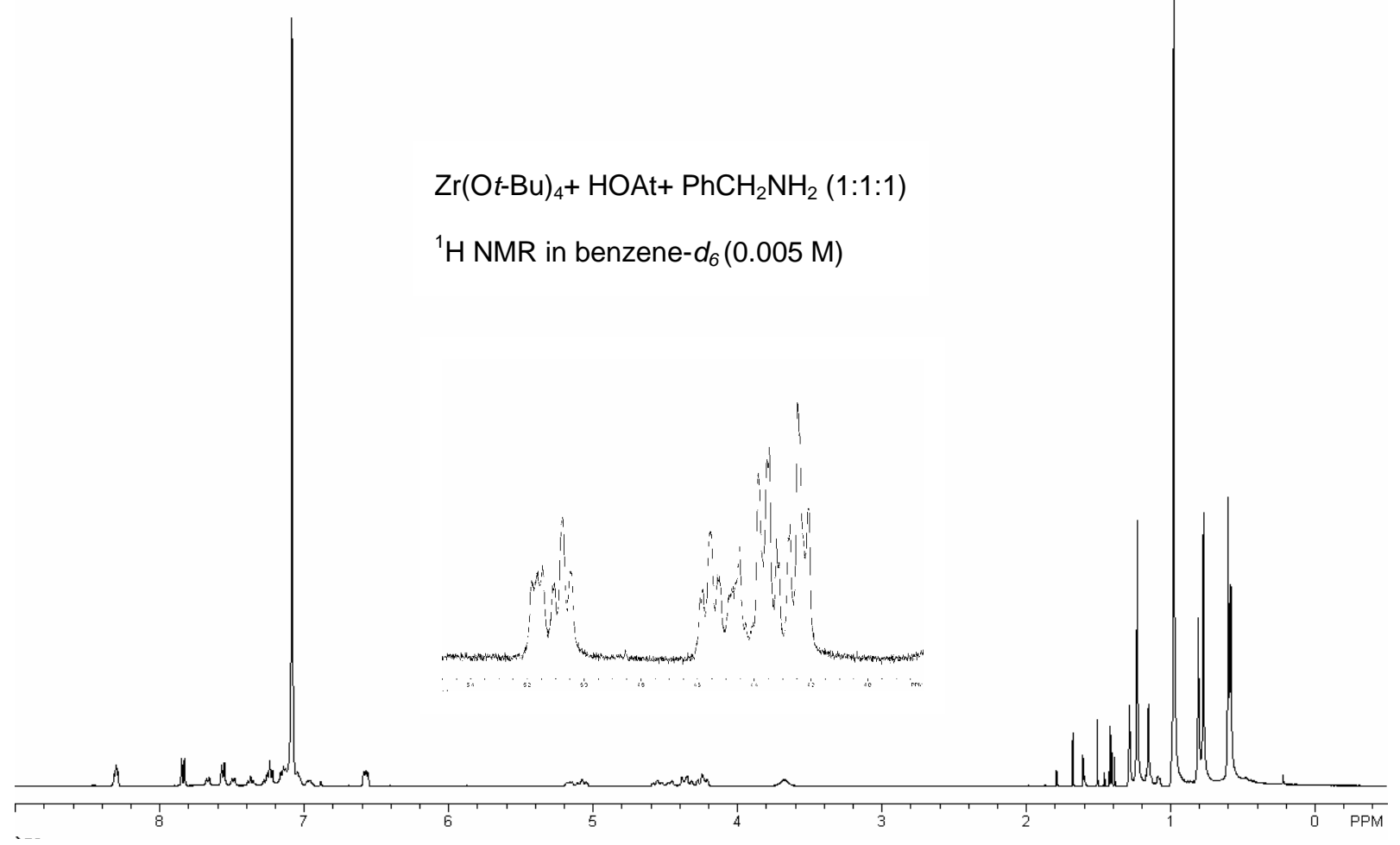

h)

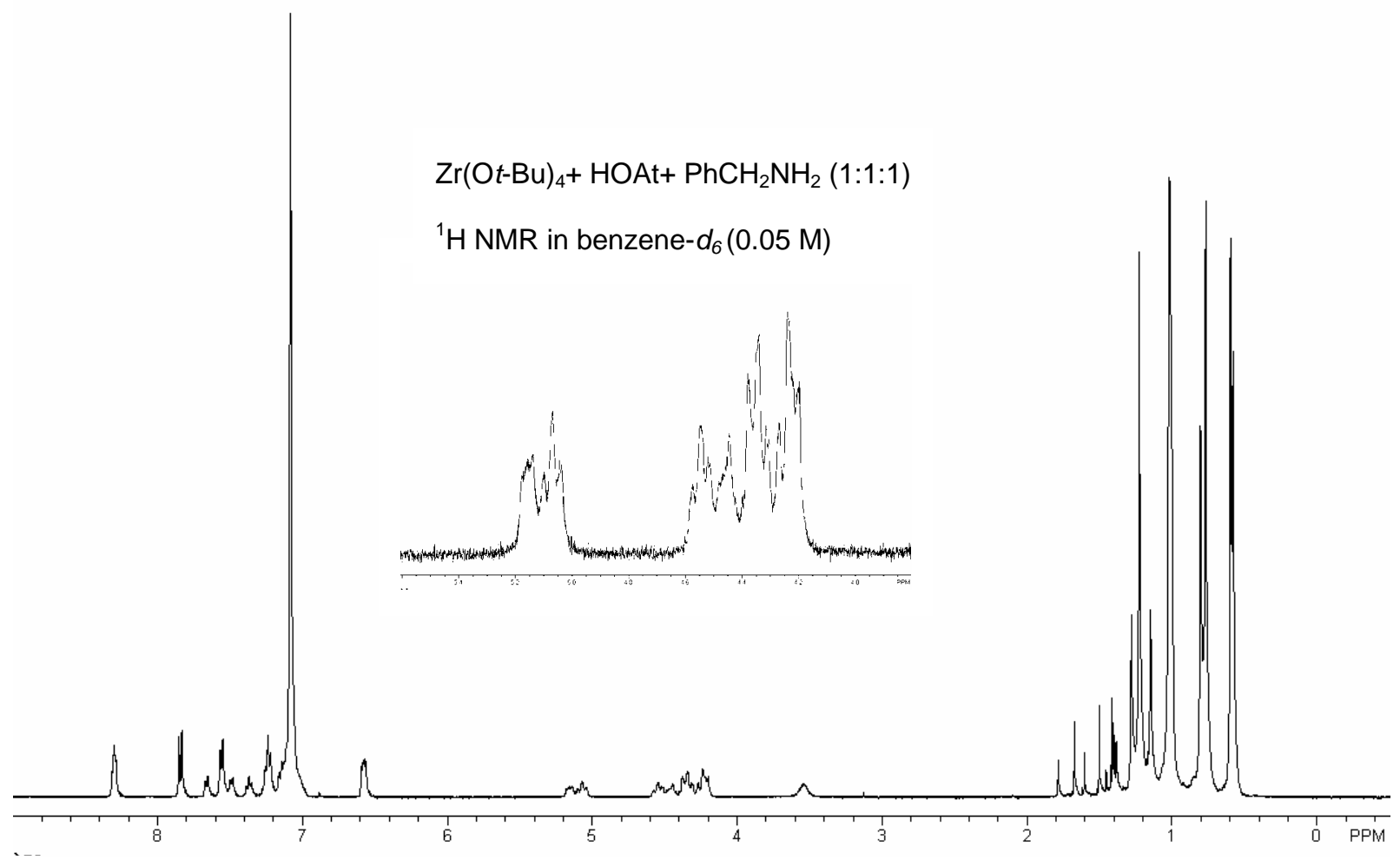



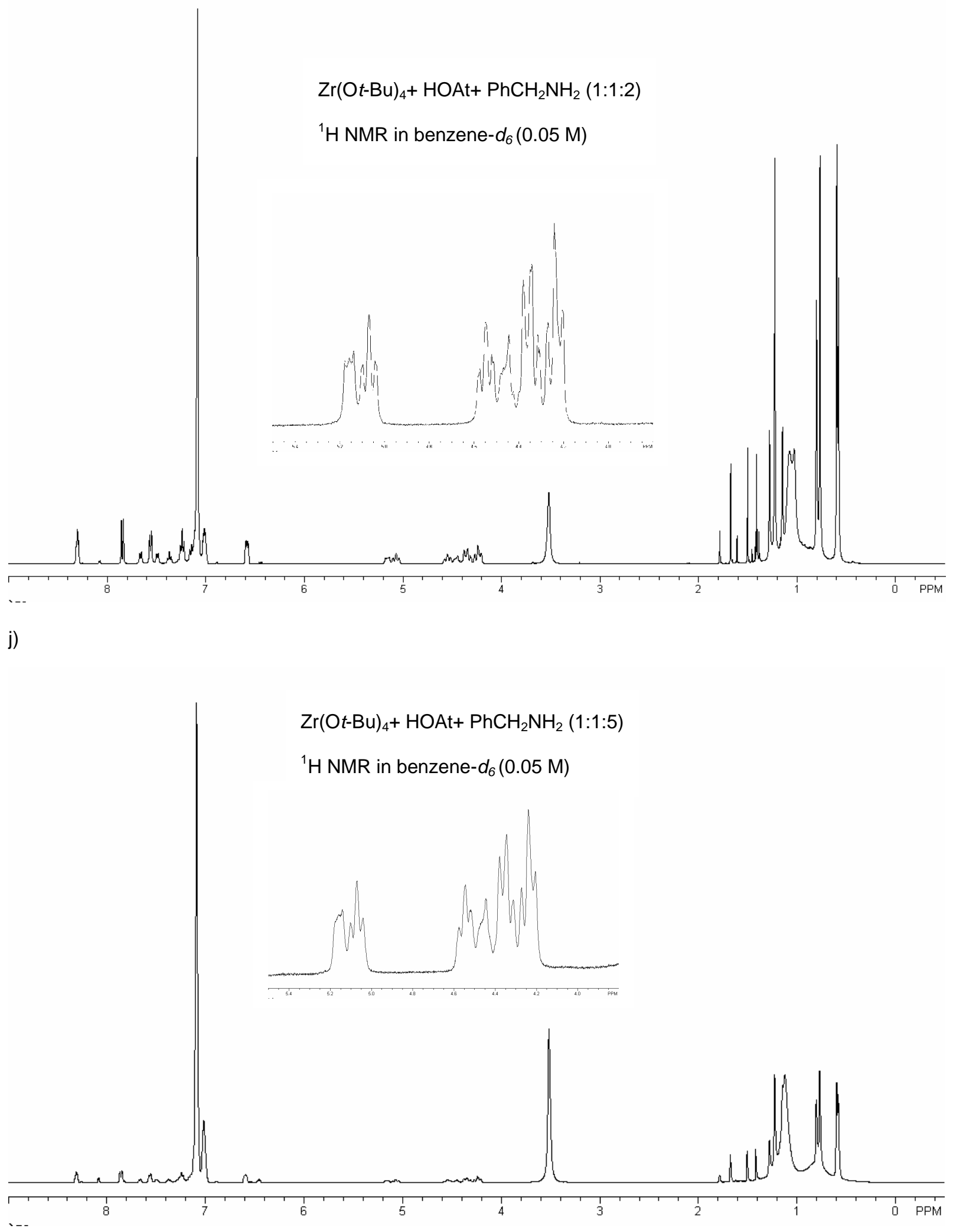
k)

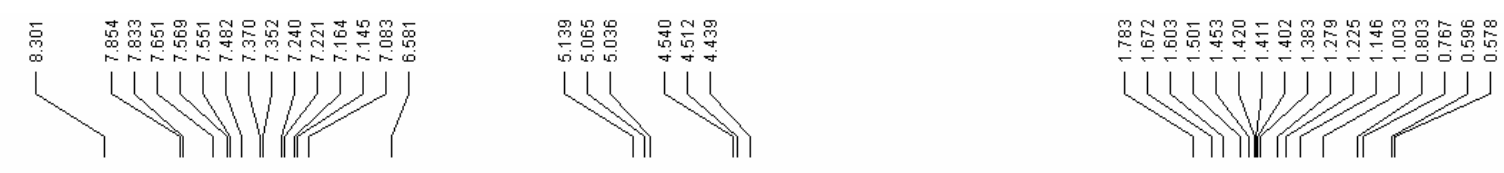

$\mathrm{Zr}(\mathrm{Ot}-\mathrm{Bu})_{4}+\mathrm{HOAt}+\mathrm{PhCD}_{2} \mathrm{NH}_{2}(1: 1: 1)$

${ }^{1} \mathrm{H}$ NMR in benzene- $d_{6}(0.05 \mathrm{M})$

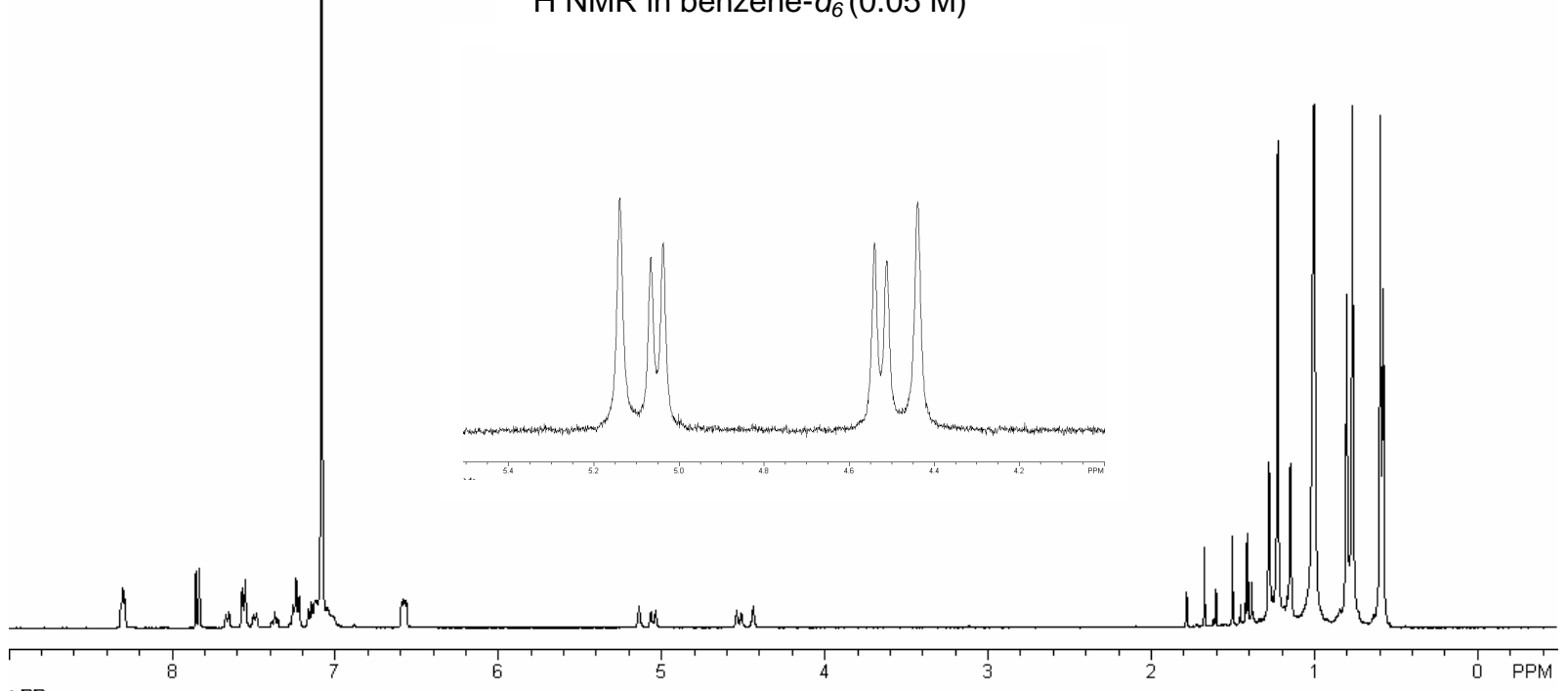

nn

l)

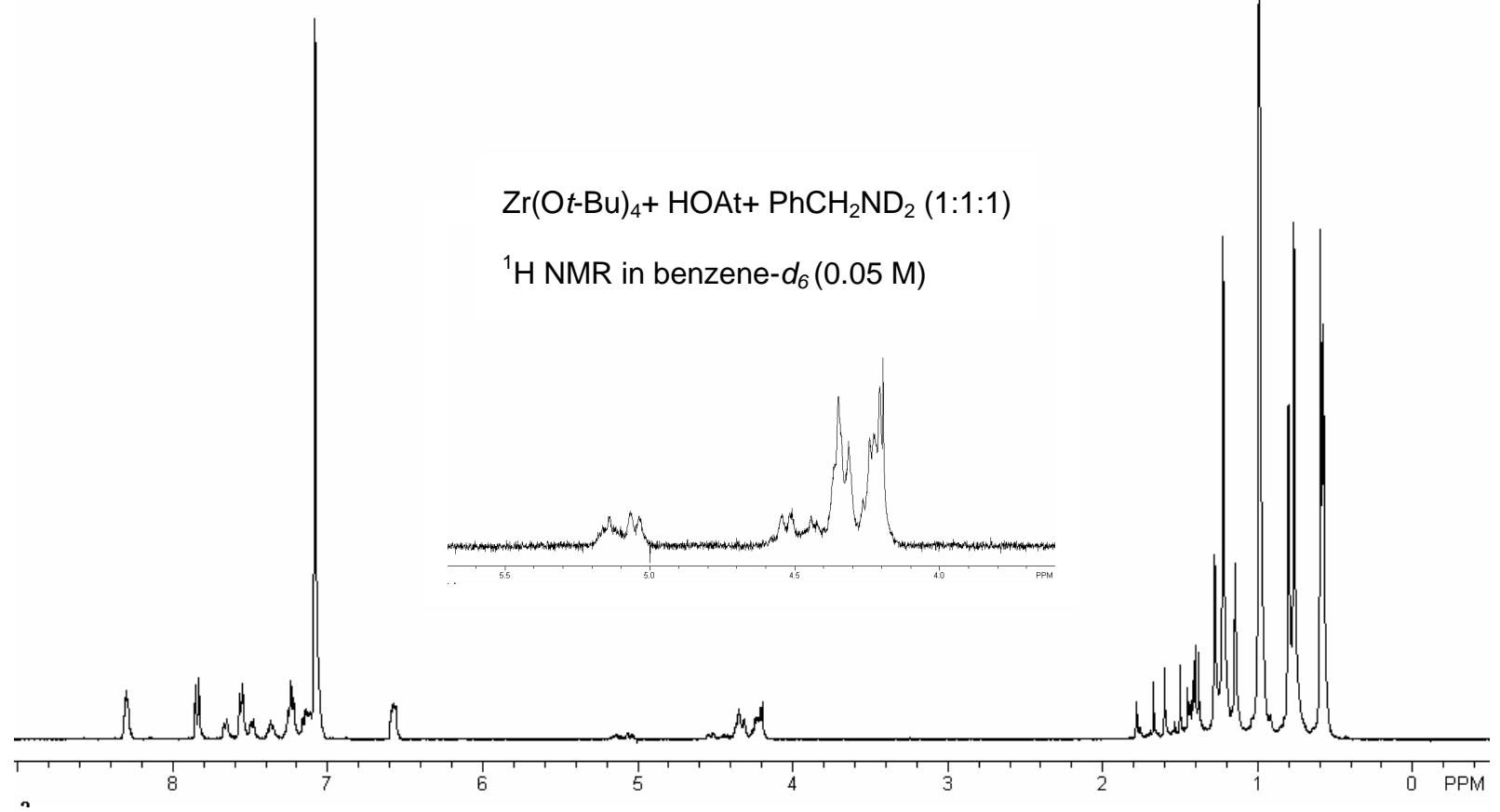


m)

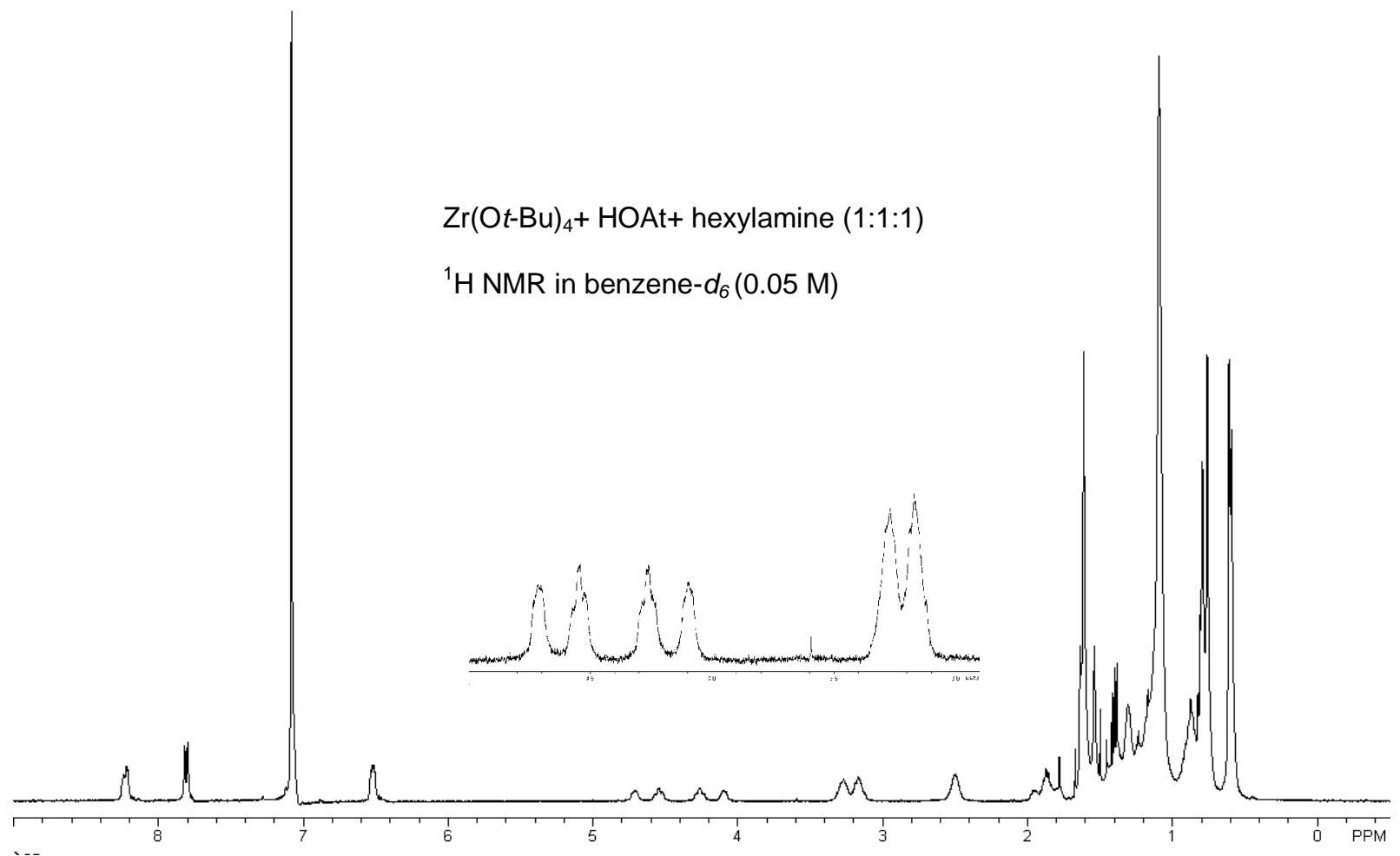

n)

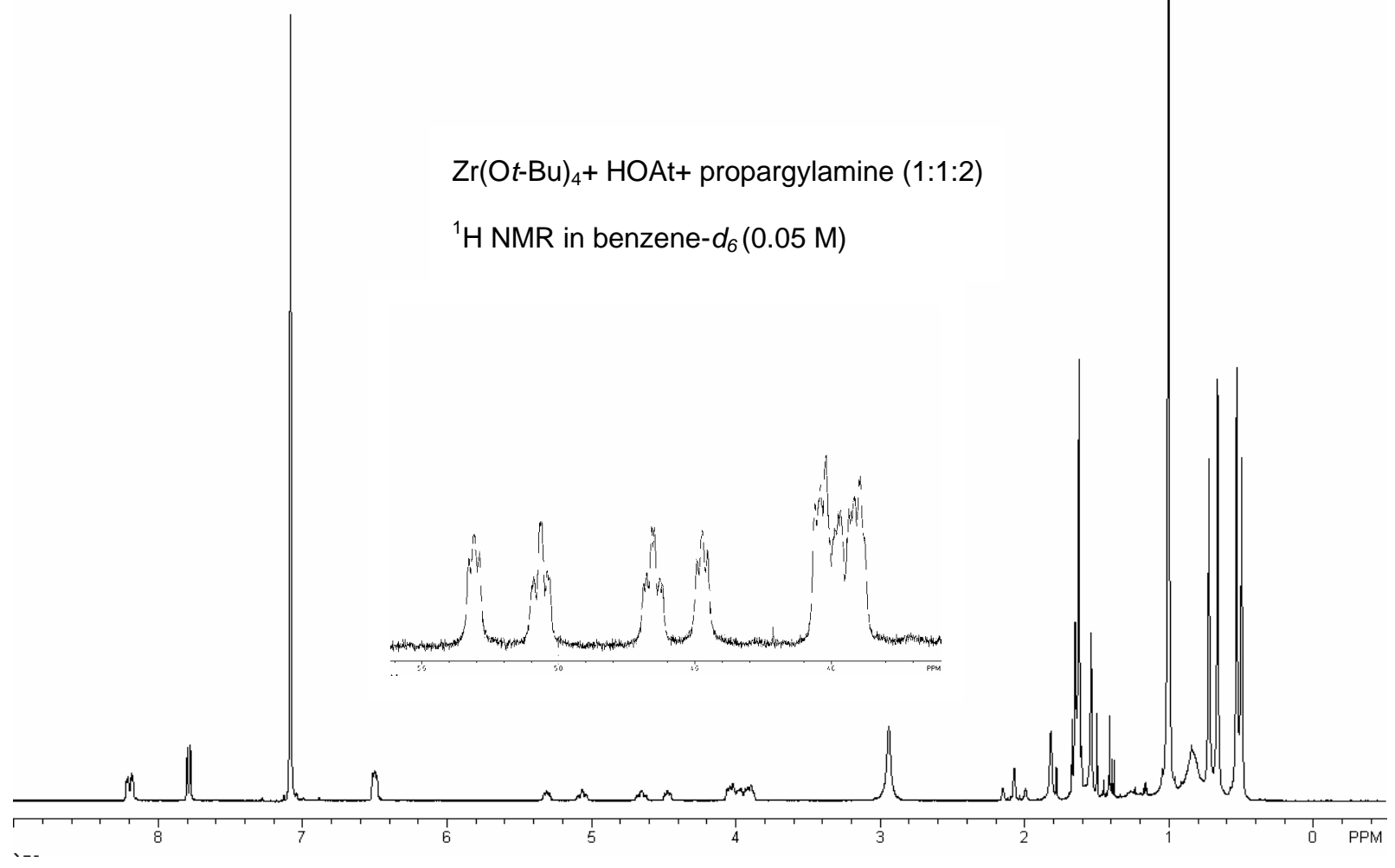


o)

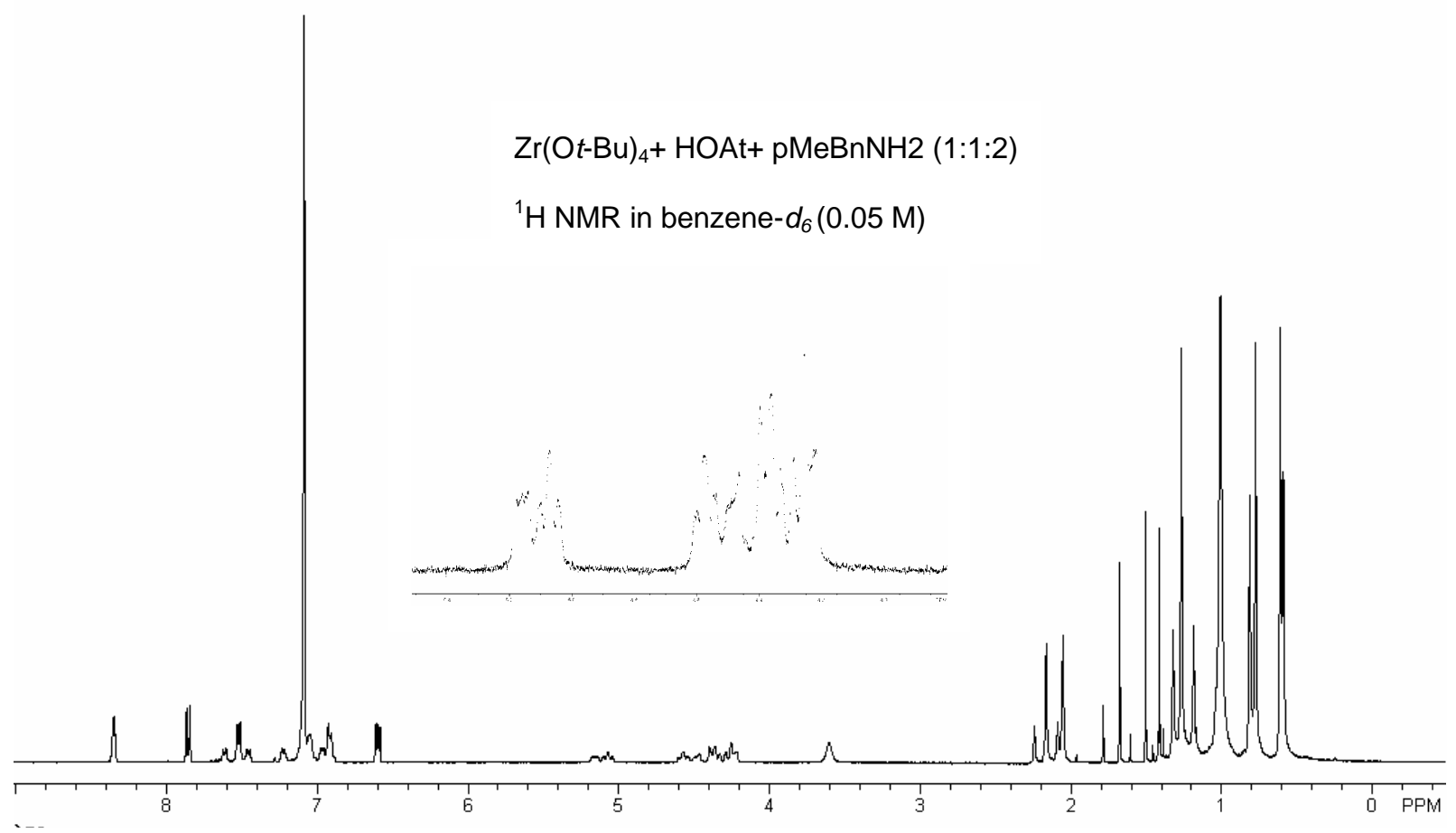

p)

$\mathrm{Zr}(\mathrm{Ot}-\mathrm{Bu})_{4}+\mathrm{HOAt}+\mathrm{PhCH}_{2} \mathrm{NH}_{2}+\mathrm{MeOH}(1: 1: 1: 1)$

${ }^{1} \mathrm{H}$ NMR in benzene- $d_{6}(0.05 \mathrm{M})$

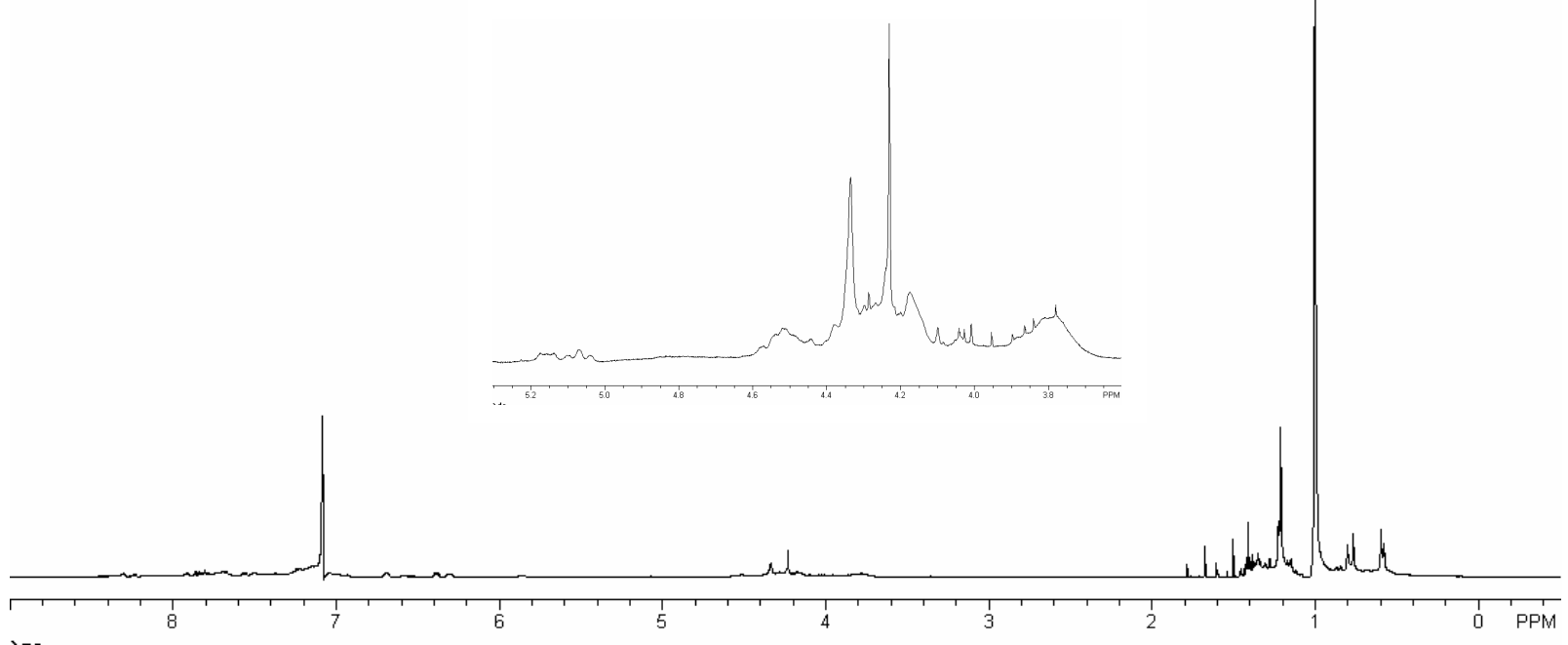


q)

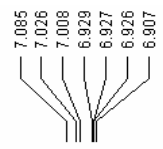

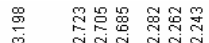
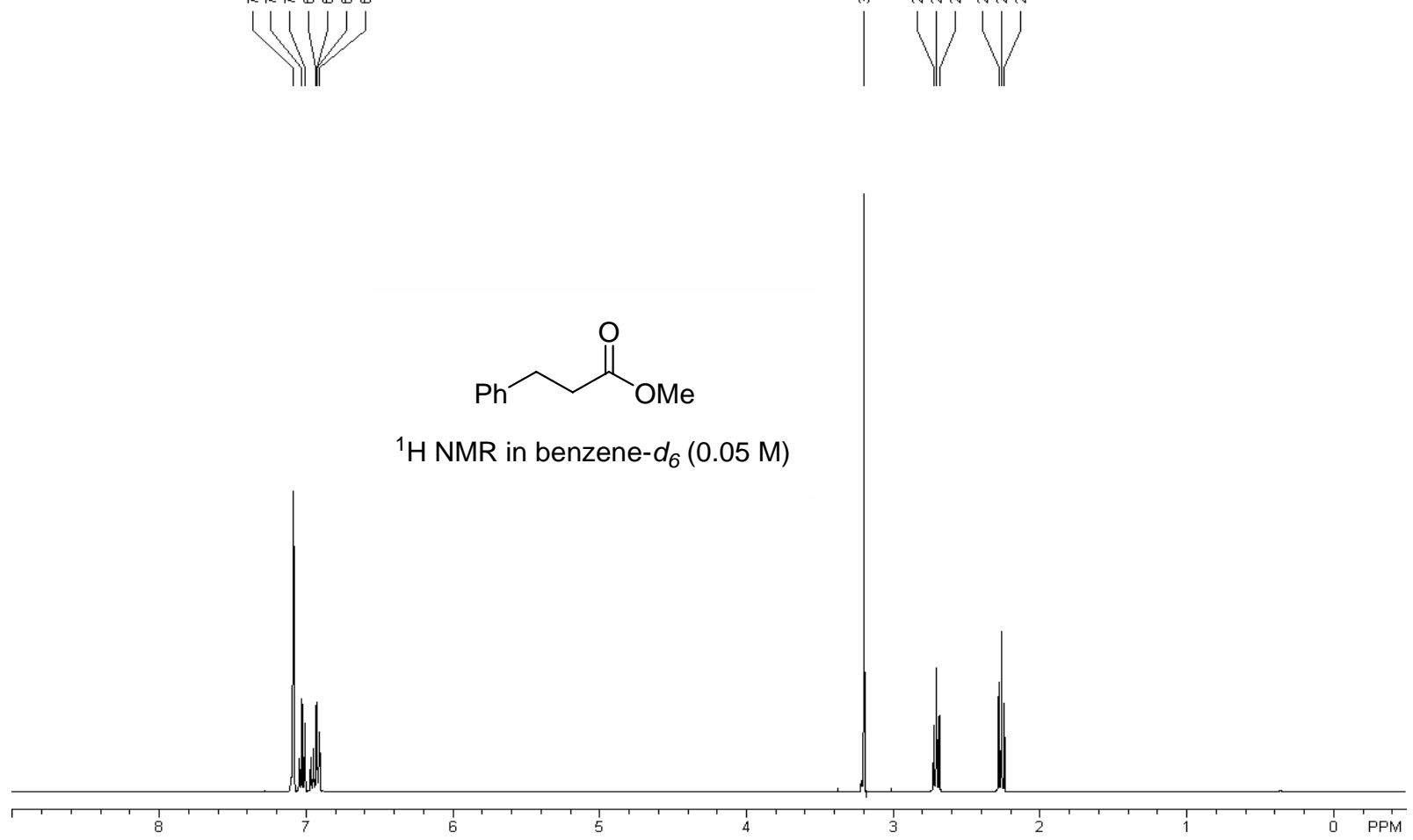

r)
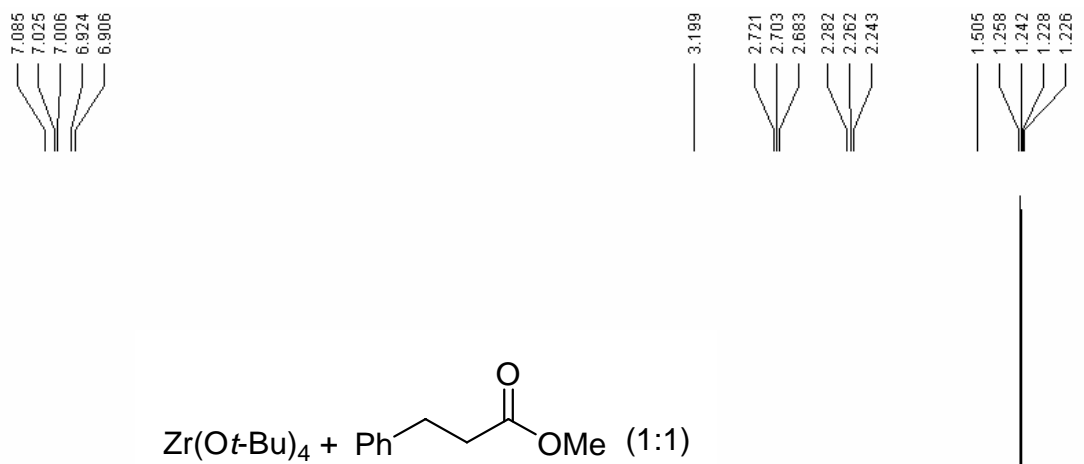

${ }^{1} \mathrm{H}$ NMR in benzene- $d_{6}(0.05 \mathrm{M})$

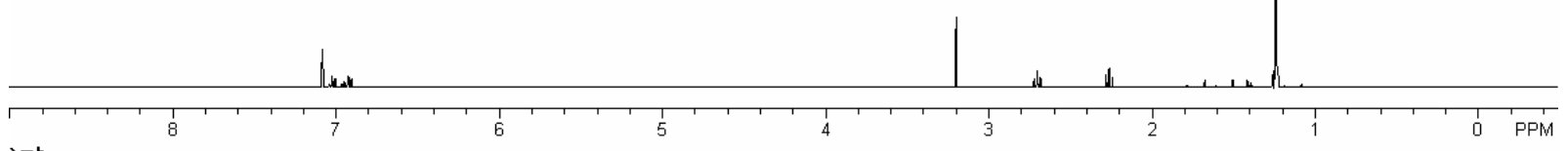


s)

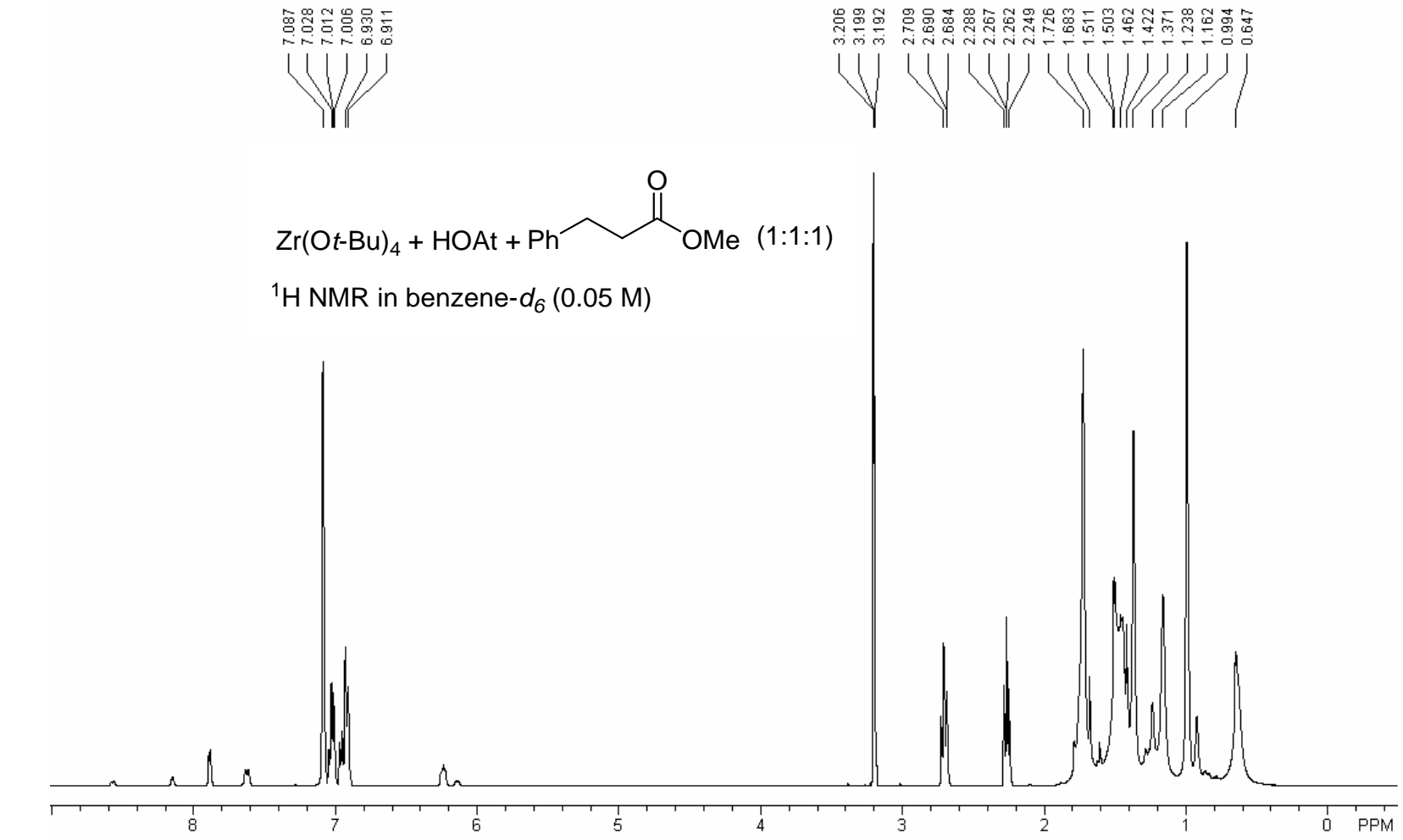

t)
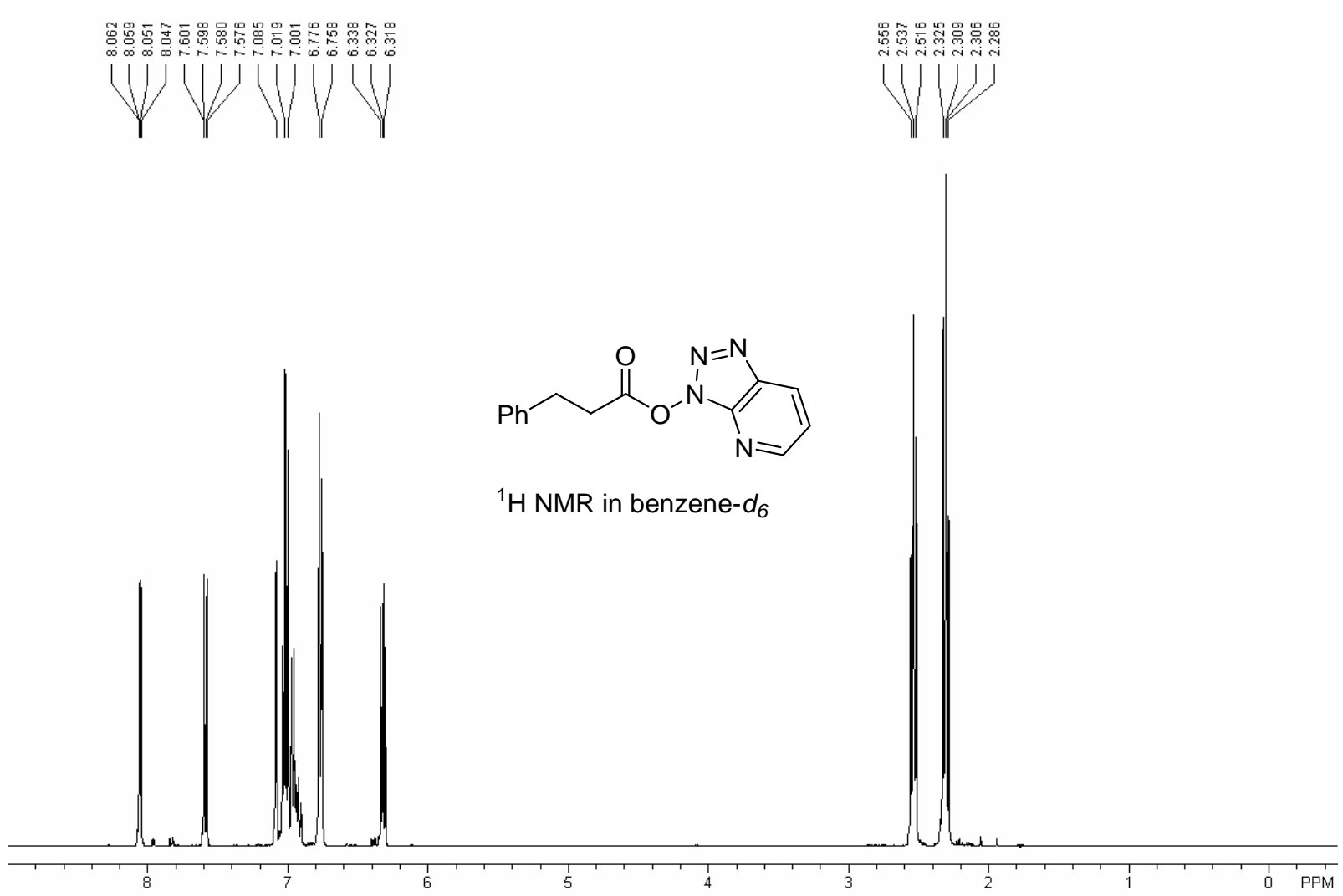
2. HOBt or HYP as additive.

a)

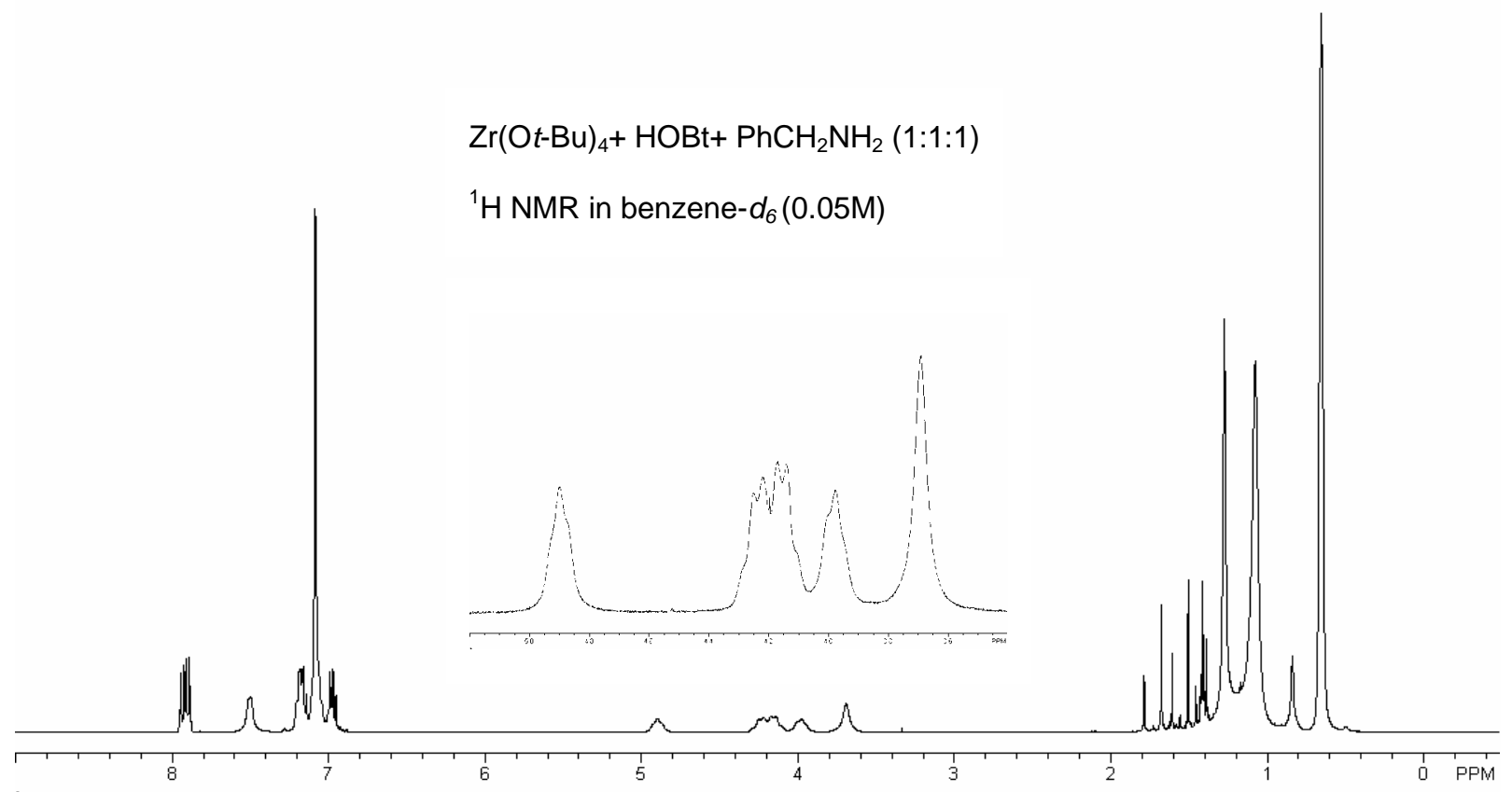

b)

$\mathrm{Zr}(\mathrm{Ot}-\mathrm{Bu})_{4}+\mathrm{HYP}+\mathrm{PhCH}_{2} \mathrm{NH}_{2}(1: 1: 1)$

${ }^{1} \mathrm{H}$ NMR in benzene- $d_{6}(0.05 \mathrm{M})$

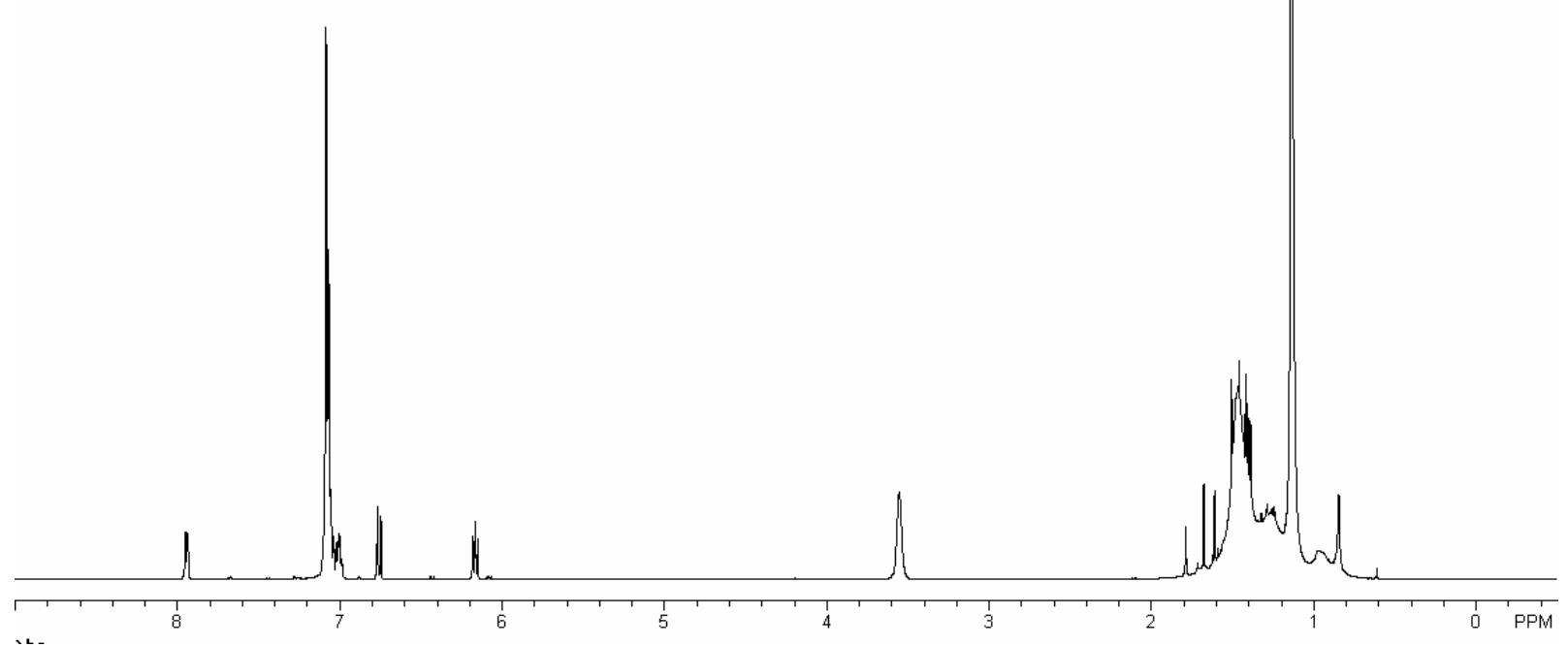


3. Reaction monitoring.

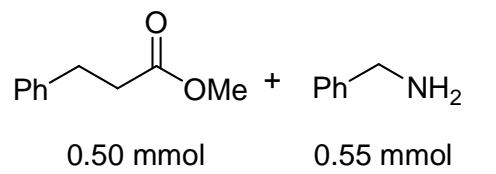

$10 \mathrm{~mol} \% \mathrm{Zr}(\mathrm{Ot}-\mathrm{Bu})_{4}$

$10 \mathrm{~mol} \% \mathrm{HOAt}$

toluene- $d_{8}(0.5 \mathrm{~mL})$

$\mathrm{rt}, 2 \mathrm{~h}$ then $100^{\circ} \mathrm{C}, 7 \mathrm{~h}$<smiles>O=C(CCc1ccccc1)NCc1ccccc1</smiles>

rt, 2 min, 3\% conversion

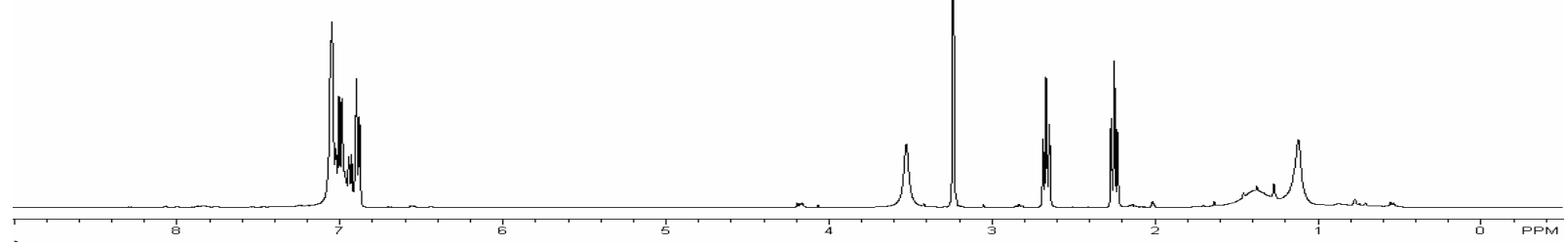

rt, 2 h, $28 \%$ conversion

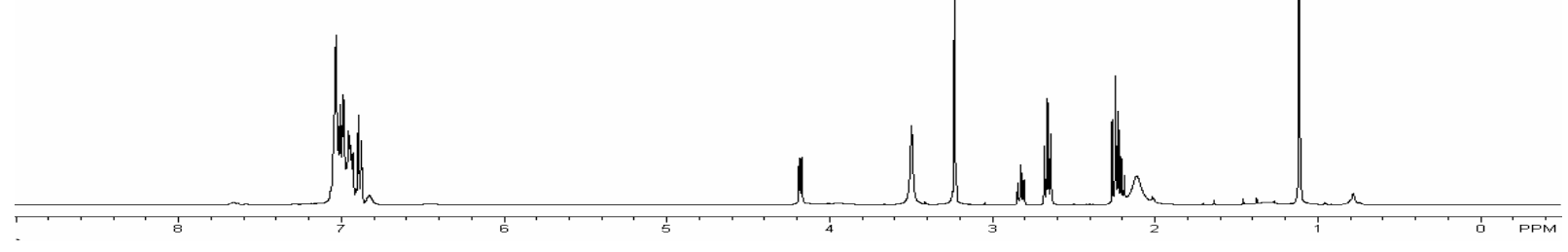

$100^{\circ} \mathrm{C}, 30 \mathrm{~min}, 66 \%$ conversion

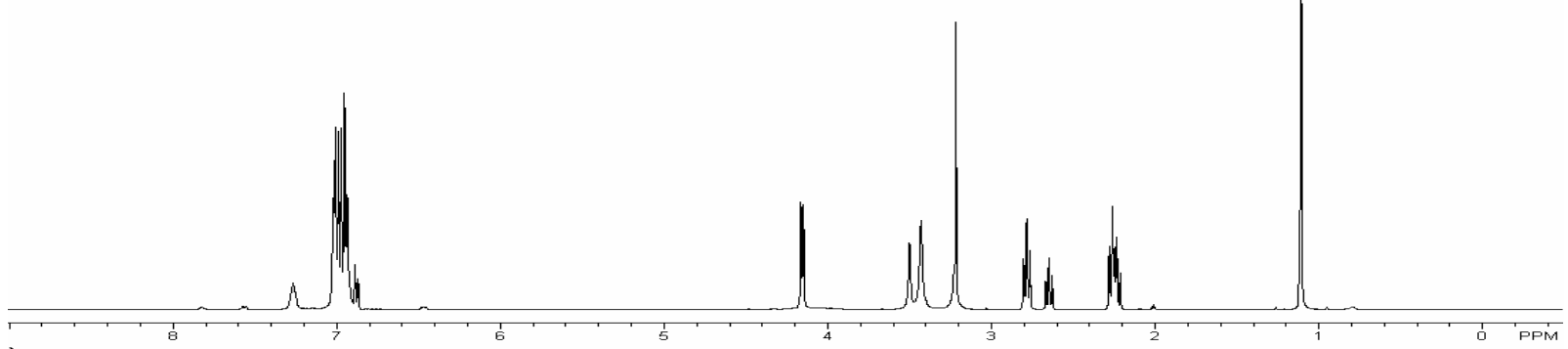


$100^{\circ} \mathrm{C}, 2 \mathrm{~h}, 83 \%$ conversion

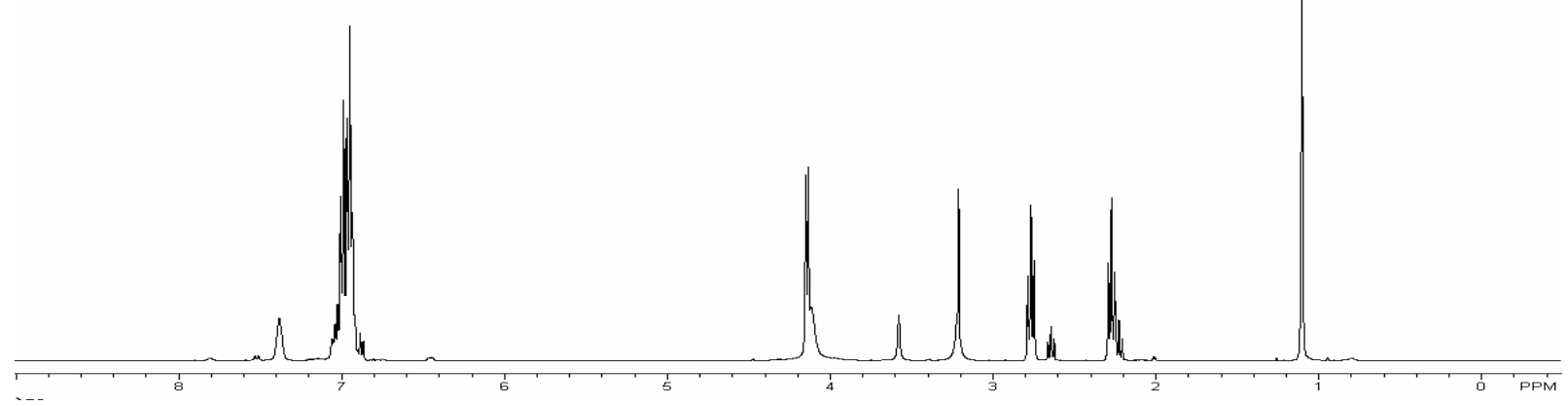

$100^{\circ} \mathrm{C}, 7 \mathrm{~h}, 92 \%$ conversion

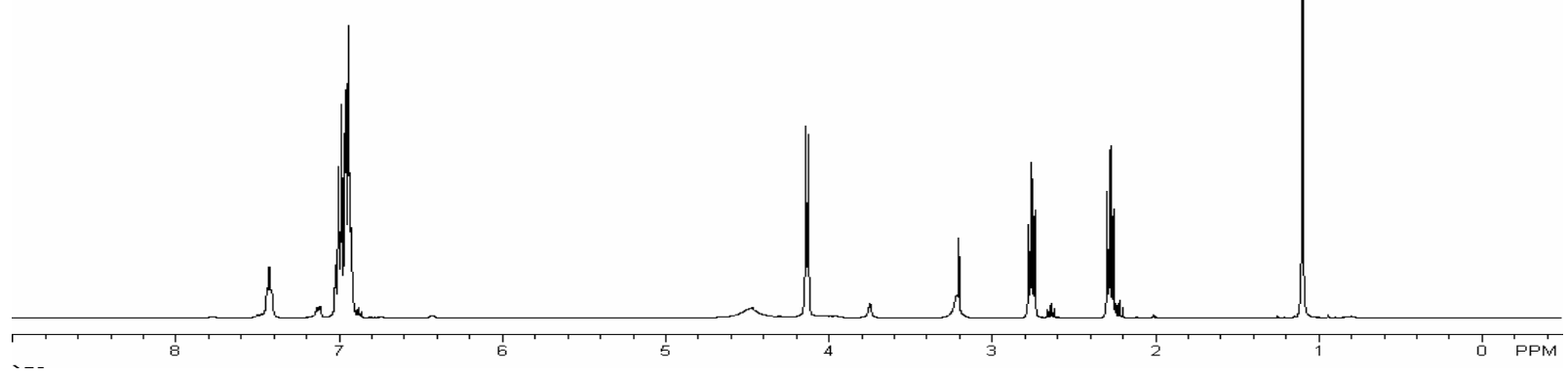

$100^{\circ} \mathrm{C}, 7 \mathrm{~h}, 92 \%$ conversion

$t-\mathrm{BuOH}$ and $\mathrm{MeOH}$ added

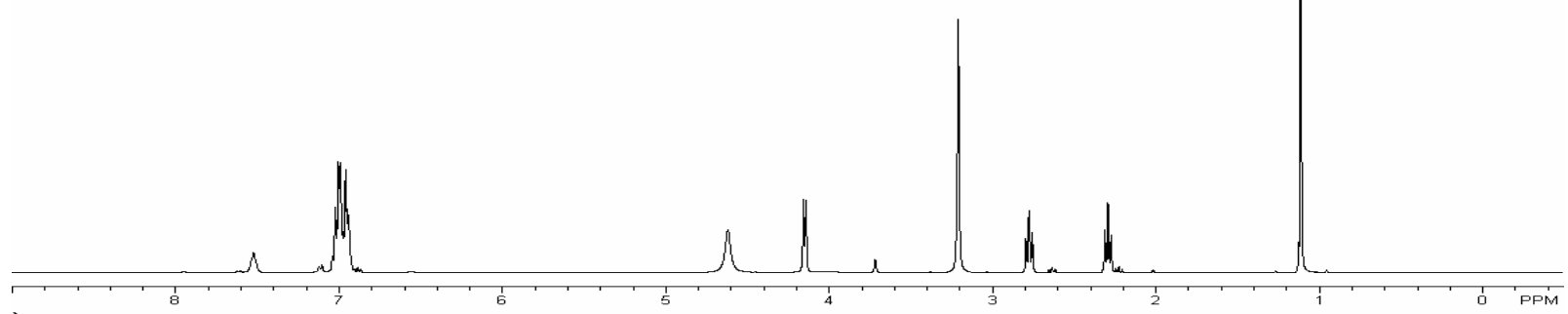




\section{Variable temperature and $2 \mathrm{D}{ }^{1} \mathrm{H}$ NMR studies:}

$\mathrm{Zr}(\mathrm{Ot}-\mathrm{Bu})_{4}$ : HOAt: $\mathrm{BnNH}_{2}(1: 1: 1)$ in $1 \mathrm{~mL}$ toluene- $d_{8}(0.05 \mathrm{M})$ varying from $-50^{\circ} \mathrm{C}$ to $85^{\circ} \mathrm{C}$.

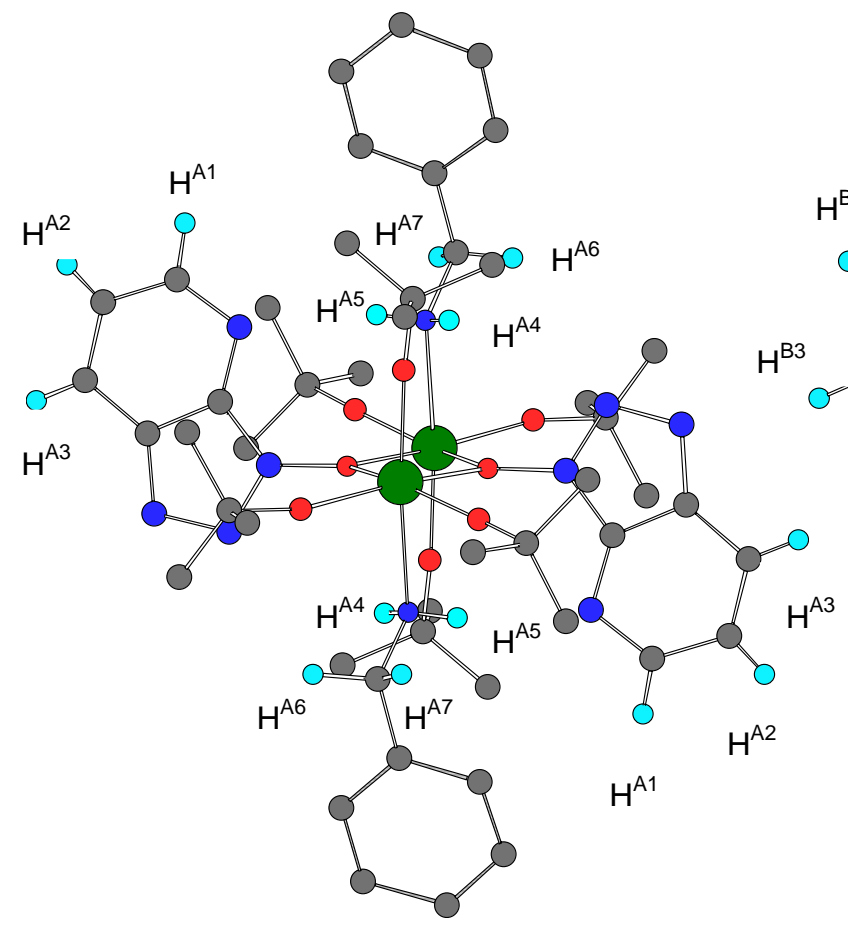

zirconium complex 8

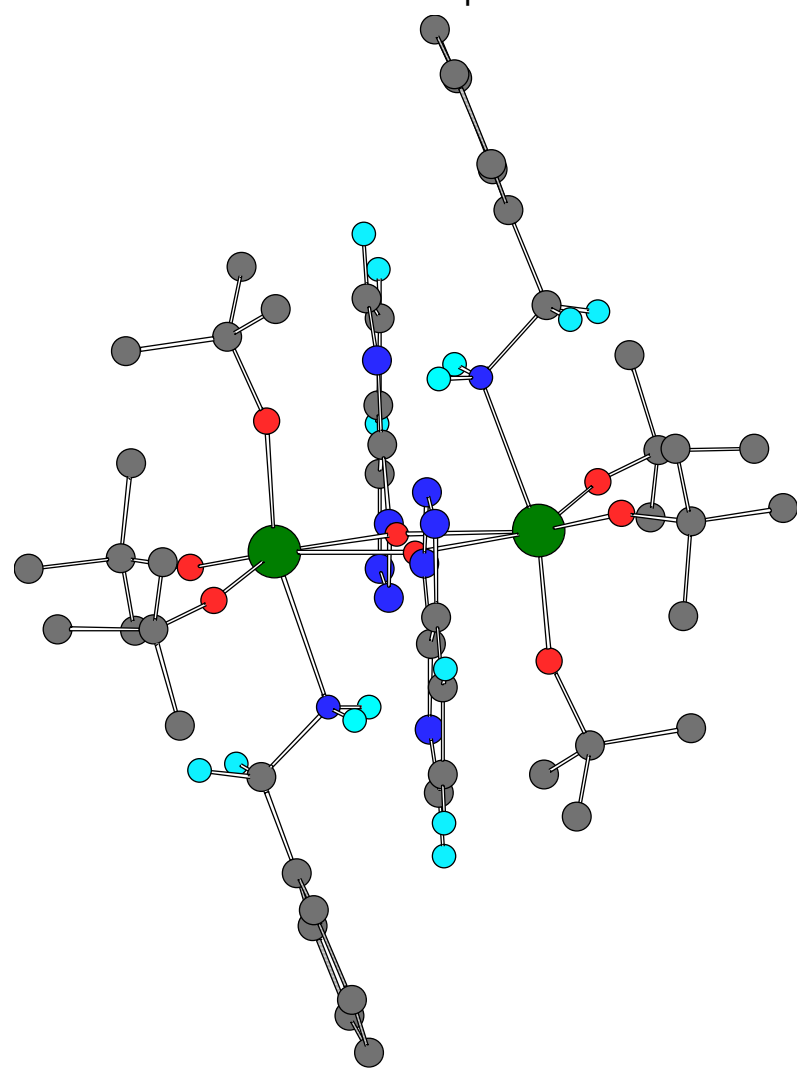

Side view of 8

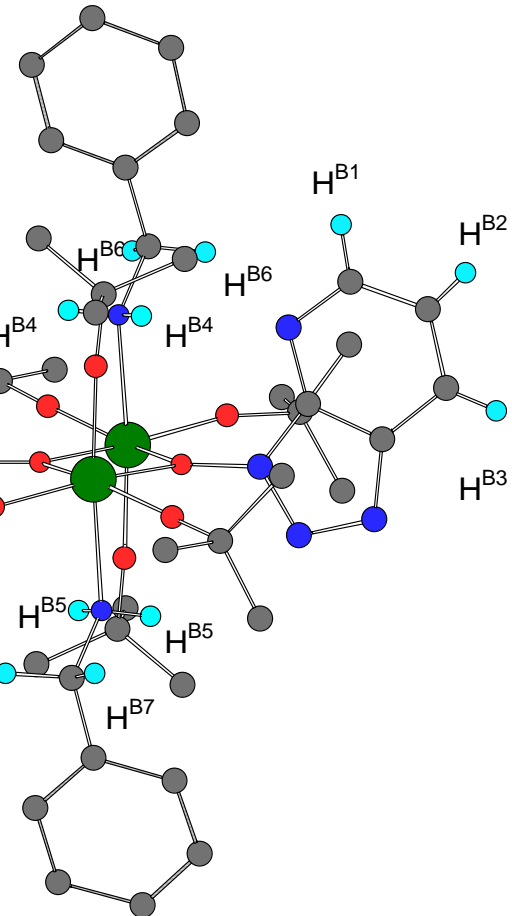

proposed conformer $\mathbf{9}$

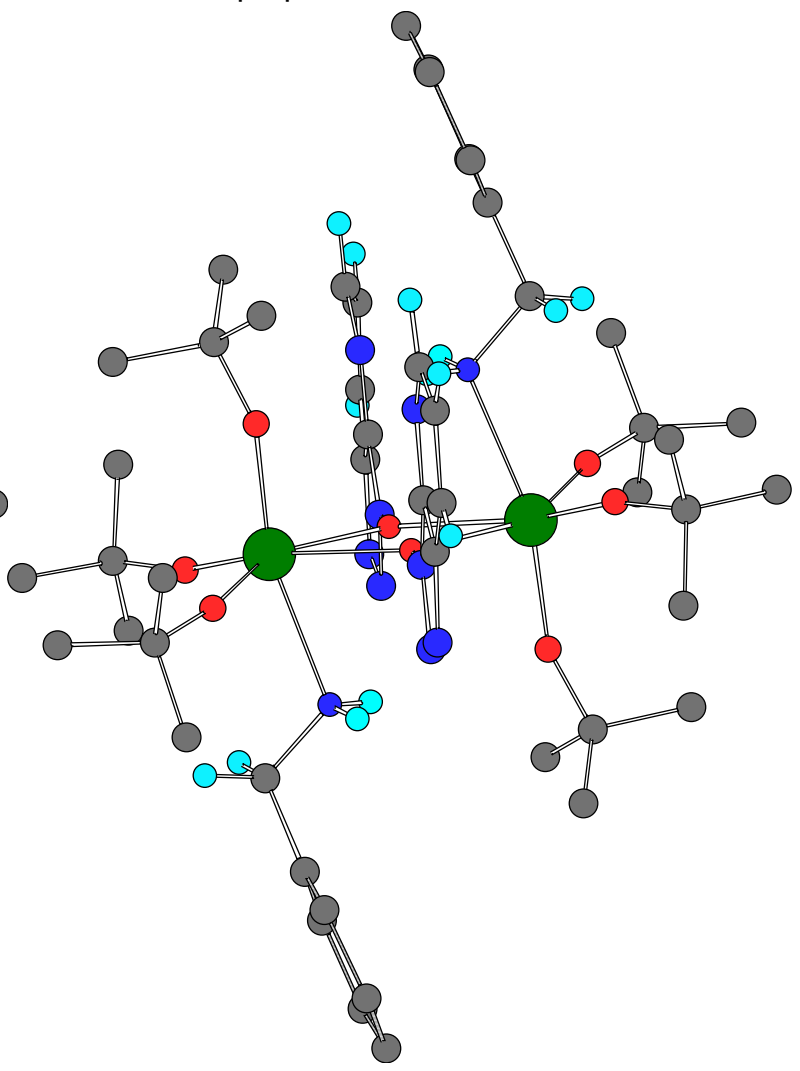

Side view of $\mathbf{9}$ 
Expanded region (6.2-8.5 ppm):
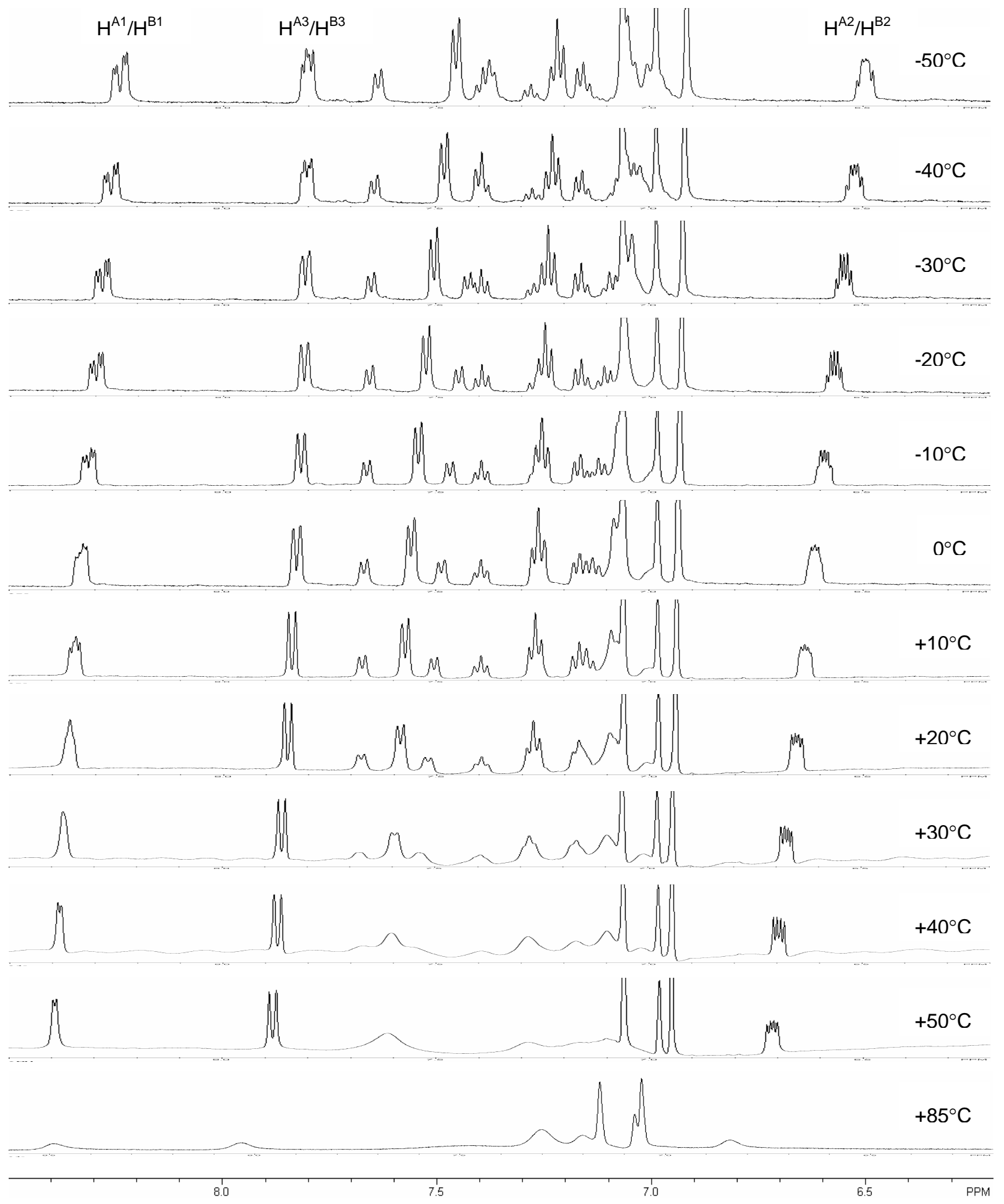

$\mathrm{S} 21$ 


\section{Expanded region (3.8-5.5 ppm):}
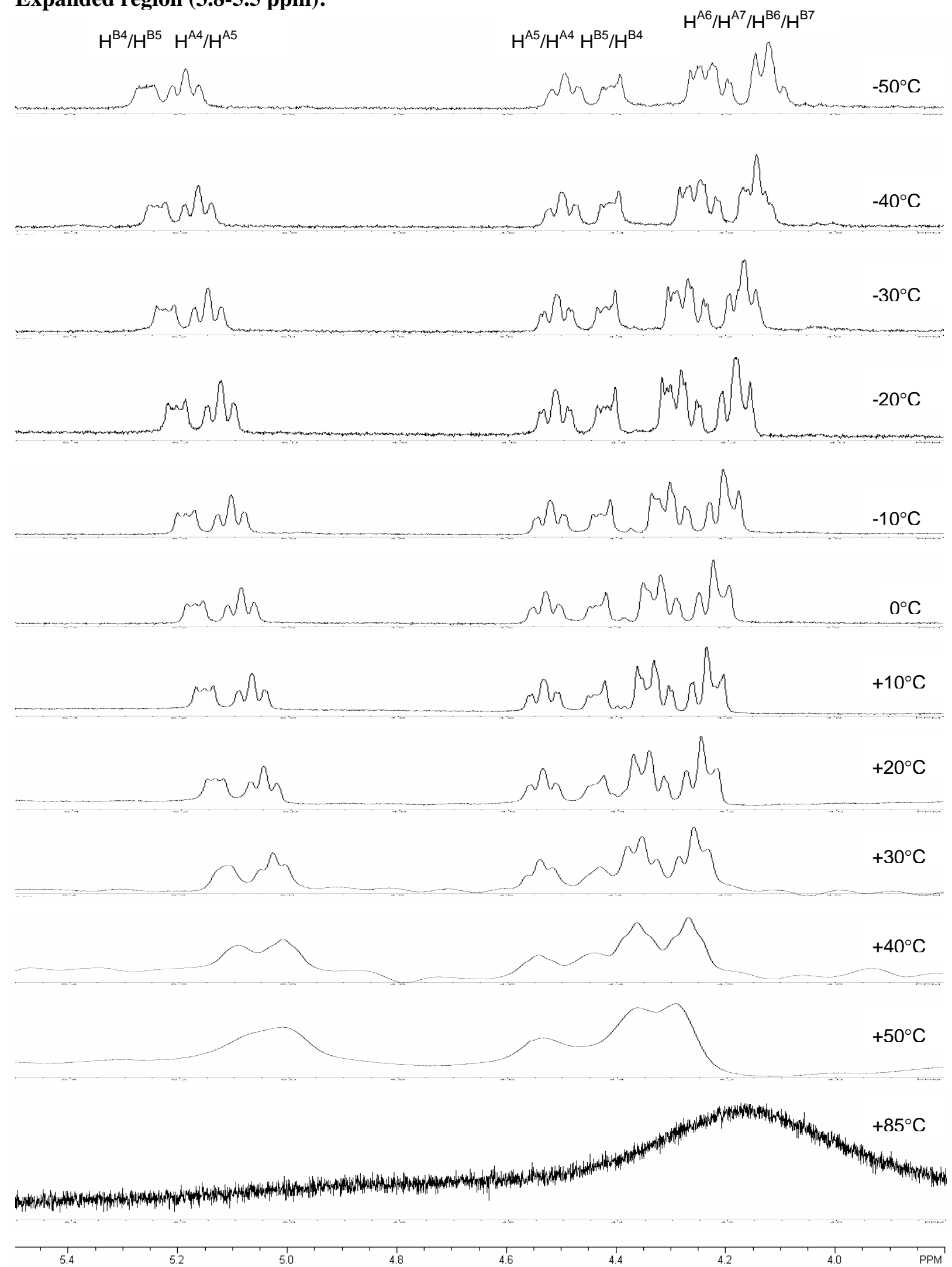
Expanded region (0.4-1.8 ppm):
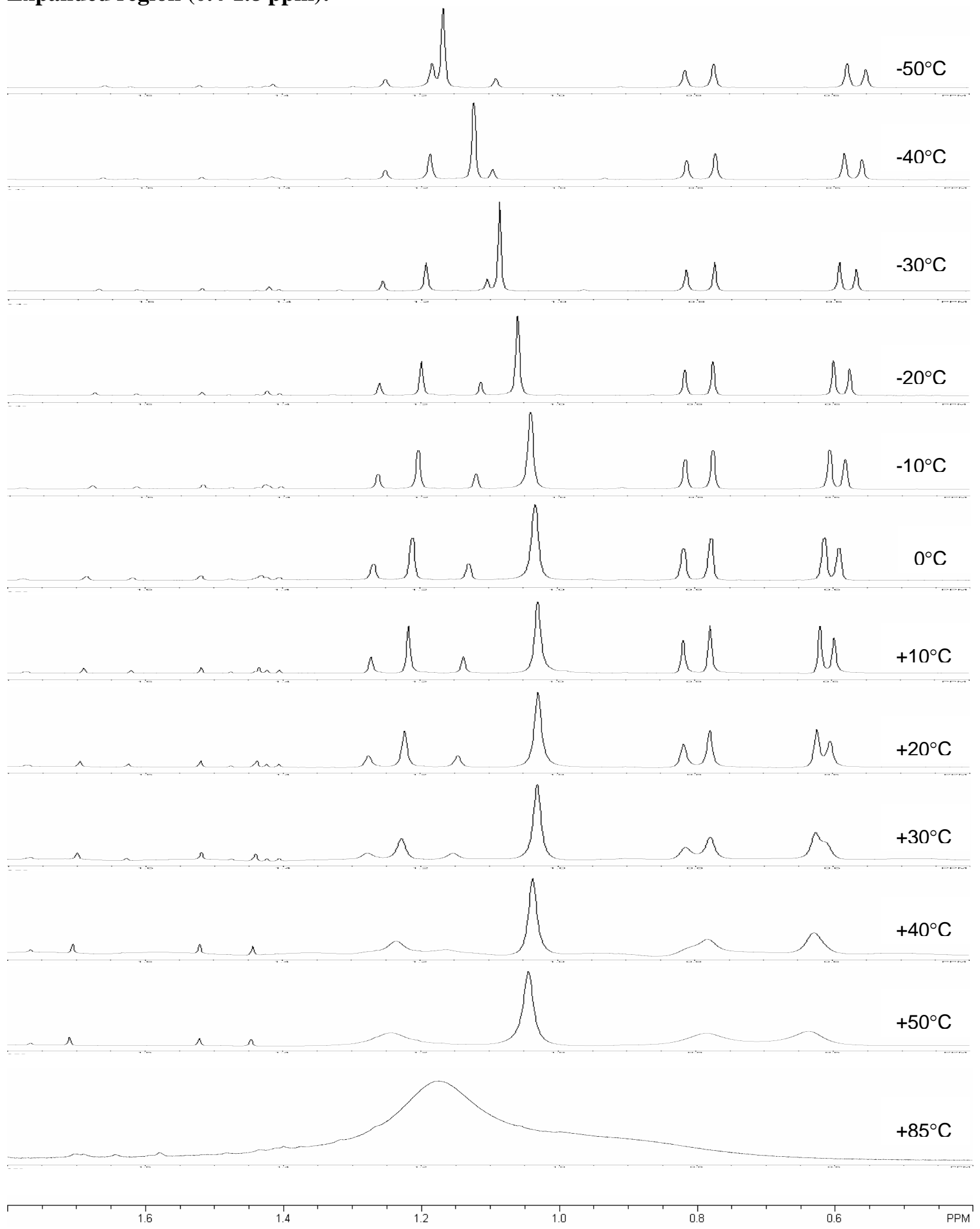

$\mathrm{S} 23$ 
$\mathrm{Zr}(\mathrm{Ot}-\mathrm{Bu})_{4}+\mathrm{HOAt}+\mathrm{PhCD}_{2} \mathrm{NH}_{2}(1: 1: 1)$ in toluene- $d_{8}(0.05 \mathrm{M})$

T-ROESY spectrum (4.4- $5.3 \mathrm{ppm})$ at $30^{\circ} \mathrm{C}$
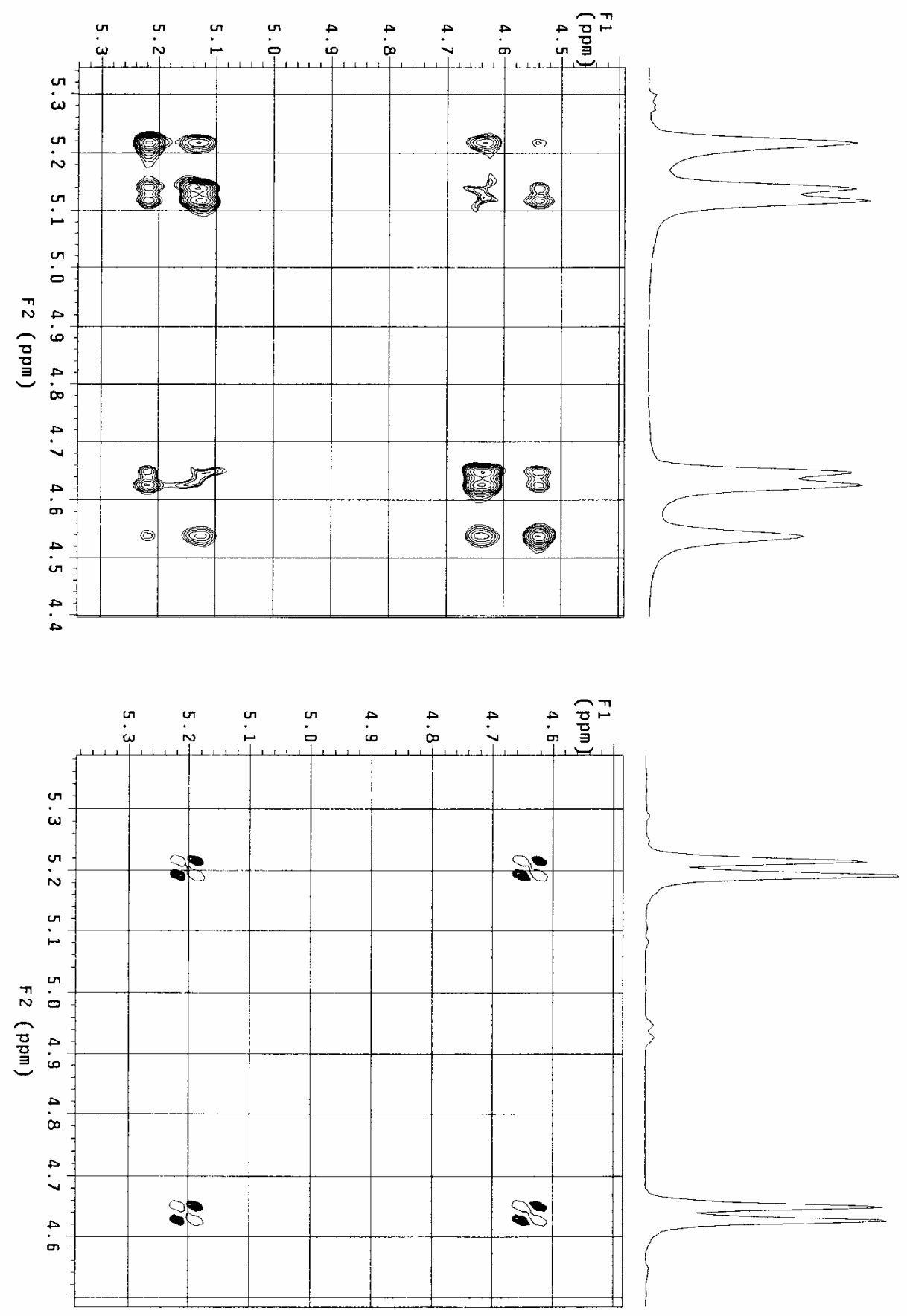

$\mathrm{Zr}(\mathrm{Ot}-\mathrm{Bu})_{4}+\mathrm{HOAt}+\mathrm{PhCD}_{2} \mathrm{NH}_{2}(1: 1: 1)$ in toluene- $d_{8}(0.05 \mathrm{M})$

DQF-COSY spectrum (4.5- $5.4 \mathrm{ppm}$ ) at $0^{\circ} \mathrm{C}$ 
VII. X-ray crystal structure of complex 8:

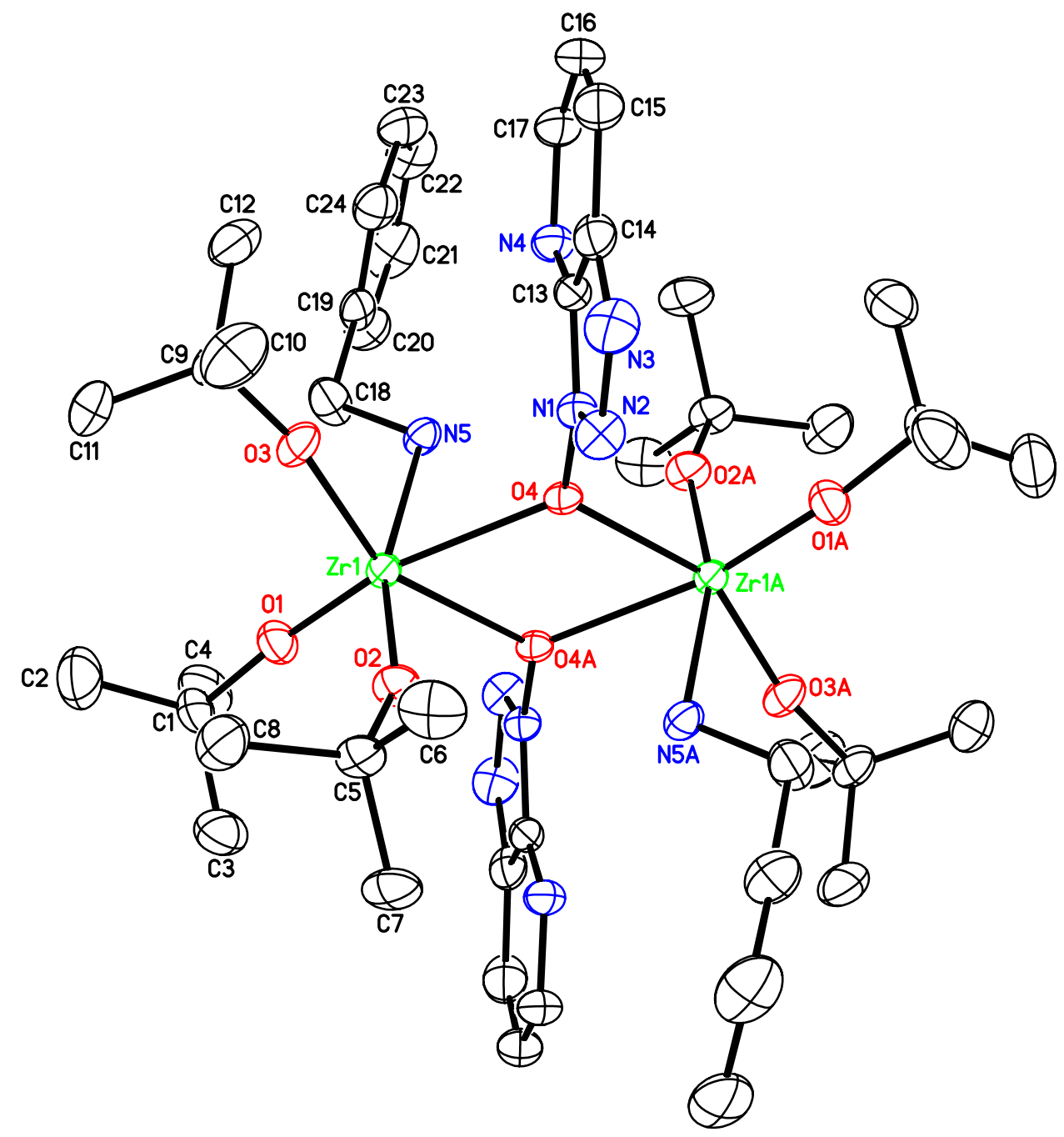

Crystals of complex 8 suitable for X-ray diffraction analysis were obtained by slow evaporation from benzene- $d_{6}$. Crystallographic data have been deposited with the Cambridge Crystallographic Data Centre (CCDC 267260). Copies of the data can be obtained free of charge on application to the CCDC, 12 Union Road, Cambridge CB21EZ, UK (fax: (+44)-1223-336-033; e-mail: deposit@ccdc.cam.ac.uk. 
Table 1. Crystal data and structure refinement for complex 8.

Identification code

Empirical formula

Formula weight

Temperature

Wavelength

Crystal system

Space group

Unit cell dimensions

Volume

Z

Density (calculated)

Absorption coefficient

$\mathrm{F}(000)$

Crystal size

Theta range for data collection

Index ranges

Reflections collected

Independent reflections

Completeness to theta $=25.23^{\circ}$

Absorption correction

Max. and min. transmission

Refinement method

Data / restraints / parameters

Goodness-of-fit on $\mathrm{F}^{2}$

Final $\mathrm{R}$ indices $[\mathrm{I}>2 \operatorname{sigma}(\mathrm{I})]$

$\mathrm{R}$ indices (all data)

Largest diff. peak and hole
Complex 8

C30 H37 D8 N5 O4 Zr

638.98

173(2) K

$0.71073 \AA$

Monoclinic

$\mathrm{P} 2(1) / \mathrm{c}$

$\begin{array}{ll}\mathrm{a}=12.3129(3) \AA & \alpha=90^{\circ} . \\ \mathrm{b}=12.9150(3) \AA & \beta=100.849(1)^{\circ} . \\ \mathrm{c}=21.3187(5) \AA & \gamma=90^{\circ} .\end{array}$

$3329.53(14) \AA^{3}$

4

$1.275 \mathrm{Mg} / \mathrm{m}^{3}$

$0.369 \mathrm{~mm}^{-1}$

1328

$0.30 \times 0.15 \times 0.02 \mathrm{~mm}^{3}$

1.68 to $25.23^{\circ}$.

$-13<=\mathrm{h}<=14,-15<=\mathrm{k}<=14,-25<=1<=25$

25543

$6021[\mathrm{R}($ int $)=0.0426]$

$100.0 \%$

Semiempirical from equivalents

0.9927 and 0.8974

Full-matrix least-squares on $\mathrm{F}^{2}$

$6021 / 0 / 517$

1.022

$\mathrm{R} 1=0.0304, \mathrm{wR} 2=0.0684$

$\mathrm{R} 1=0.0490, \mathrm{wR} 2=0.0741$

0.423 and -0.438 e. $\AA^{-3}$ 
Table 2. Atomic coordinates $\left(\times 10^{4}\right)$ and equivalent isotropic displacement parameters $\left(\AA^{2} \times 10^{3}\right)$ for complex 8 . $\mathrm{U}(\mathrm{eq})$ is defined as one third of the trace of the orthogonalized $\mathrm{U}^{\mathrm{ij}}$ tensor.

\begin{tabular}{|c|c|c|c|c|}
\hline & $\mathrm{x}$ & $\mathrm{y}$ & $\mathrm{z}$ & $\mathrm{U}(\mathrm{eq})$ \\
\hline $\operatorname{Zr}(1)$ & $6400(1)$ & 9281(1) & $43(1)$ & $21(1)$ \\
\hline $\mathrm{O}(1)$ & 7593(1) & $9326(1)$ & $762(1)$ & $34(1)$ \\
\hline $\mathrm{O}(2)$ & $5707(1)$ & 7972(1) & $178(1)$ & $31(1)$ \\
\hline $\mathrm{O}(3)$ & $7067(1)$ & $8966(1)$ & $-681(1)$ & $33(1)$ \\
\hline $\mathrm{O}(4)$ & $4768(1)$ & $9827(1)$ & $-566(1)$ & $22(1)$ \\
\hline $\mathrm{N}(1)$ & $4531(1)$ & $9609(1)$ & $-1197(1)$ & $23(1)$ \\
\hline $\mathrm{N}(2)$ & $3974(2)$ & $8749(2)$ & $-1412(1)$ & $33(1)$ \\
\hline $\mathrm{N}(3)$ & $3853(2)$ & $8743(2)$ & $-2042(1)$ & $41(1)$ \\
\hline $\mathrm{N}(4)$ & $5333(2)$ & $11085(2)$ & $-1634(1)$ & $31(1)$ \\
\hline $\mathrm{N}(5)$ & $6754(2)$ & $11065(2)$ & $-188(1)$ & $27(1)$ \\
\hline $\mathrm{C}(1)$ & $8422(2)$ & $9097(2)$ & $1306(1)$ & $36(1)$ \\
\hline$C(2)$ & $9341(3)$ & $8508(4)$ & 1094(2) & $64(1)$ \\
\hline$C(3)$ & 7891(3) & $8458(3)$ & $1766(2)$ & $53(1)$ \\
\hline$C(4)$ & $8855(3)$ & $10115(3)$ & $1616(2)$ & $54(1)$ \\
\hline $\mathrm{C}(5)$ & $5577(2)$ & $6879(2)$ & $202(1)$ & $32(1)$ \\
\hline$C(6)$ & $4718(3)$ & $6563(2)$ & $-372(2)$ & $49(1)$ \\
\hline$C(7)$ & $5200(3)$ & $6608(2)$ & $819(2)$ & $44(1)$ \\
\hline $\mathrm{C}(8)$ & 6679(3) & $6378(2)$ & $185(2)$ & $53(1)$ \\
\hline $\mathrm{C}(9)$ & 7703(2) & $8618(2)$ & $-1135(1)$ & $35(1)$ \\
\hline $\mathrm{C}(10)$ & 7048(3) & 7772(3) & $-1529(2)$ & $60(1)$ \\
\hline $\mathrm{C}(11)$ & 8791(3) & $8220(3)$ & $-766(2)$ & $54(1)$ \\
\hline$C(12)$ & $7888(3)$ & $9522(3)$ & $-1554(2)$ & $54(1)$ \\
\hline $\mathrm{C}(13)$ & $4785(2)$ & $10188(2)$ & $-1678(1)$ & $26(1)$ \\
\hline $\mathrm{C}(14)$ & $4340(2)$ & $9623(2)$ & $-2219(1)$ & $35(1)$ \\
\hline$C(15)$ & $4452(2)$ & $10045(3)$ & $-2811(1)$ & $50(1)$ \\
\hline$C(16)$ & $4985(2)$ & $10968(3)$ & $-2787(1)$ & $54(1)$ \\
\hline $\mathrm{C}(17)$ & $5410(2)$ & $11458(2)$ & $-2207(1)$ & $43(1)$ \\
\hline $\mathrm{C}(18)$ & 7911(2) & $11420(2)$ & $-83(2)$ & $42(1)$ \\
\hline $\mathrm{D}(18 \mathrm{~B})$ & $8330(20)$ & $10920(20)$ & $-235(13)$ & $57(9)$ \\
\hline $\mathrm{D}(18 \mathrm{~A})$ & $8220(20)$ & $11380(20)$ & $370(15)$ & $73(10)$ \\
\hline $\mathrm{C}(19)$ & $8066(2)$ & $12479(2)$ & $-357(1)$ & $31(1)$ \\
\hline$C(20)$ & $8360(2)$ & $13319(2)$ & $41(2)$ & $44(1)$ \\
\hline $\mathrm{C}(21)$ & $8474(3)$ & $14288(2)$ & $-205(2)$ & $57(1)$ \\
\hline
\end{tabular}




\begin{tabular}{lllll}
$\mathrm{C}(22)$ & $8281(3)$ & $14429(2)$ & $-854(2)$ & $61(1)$ \\
$\mathrm{C}(23)$ & $7994(3)$ & $13605(3)$ & $-1256(2)$ & $53(1)$ \\
$\mathrm{C}(24)$ & $7889(2)$ & $12631(2)$ & $-1007(1)$ & $41(1)$ \\
$\mathrm{C}(1 \mathrm{~S})$ & $9367(3)$ & $4887(3)$ & $1637(2)$ & $73(1)$ \\
$\mathrm{H}(1 \mathrm{~S})$ & 9794 & 5446 & 1523 & 88 \\
$\mathrm{C}(2 \mathrm{~S})$ & $8325(4)$ & $5038(4)$ & $1673(2)$ & $106(2)$ \\
$\mathrm{H}(2 \mathrm{~S})$ & 8007 & 5708 & 1602 & 128 \\
$\mathrm{C}(3 \mathrm{~S})$ & $7723(4)$ & $4255(7)$ & $1809(2)$ & $135(3)$ \\
$\mathrm{H}(3 \mathrm{~S})$ & 6966 & 4382 & 1818 & 162 \\
$\mathrm{C}(4 \mathrm{~S})$ & $8120(6)$ & $3260(5)$ & $1939(2)$ & $136(3)$ \\
$\mathrm{H}(4 \mathrm{~S})$ & 7674 & 2707 & 2040 & 163 \\
$\mathrm{C}(5 \mathrm{~S})$ & $9257(4)$ & $3141(3)$ & $1909(2)$ & $86(1)$ \\
$\mathrm{H}(5 \mathrm{~S})$ & 9607 & 2488 & 1995 & 104 \\
$\mathrm{C}(6 \mathrm{~S})$ & $9834(3)$ & $3953(3)$ & $1757(2)$ & $65(1)$ \\
$\mathrm{H}(6 \mathrm{~S})$ & 10592 & 3864 & 1735 & 78 \\
& & & & \\
\hline
\end{tabular}


VIII. Kinetic studies:

$\mathrm{OHMe}_{\mathrm{Oh}}^{\mathrm{O}} \overbrace{\mathrm{NH}_{2}} \stackrel{\text { cat. } \mathrm{Zr}(\mathrm{Ot}-\mathrm{Bu})_{4} \text {-HOAt }}{\text { toluene, } \mathrm{rt}}$

Rate order to amine concentration:

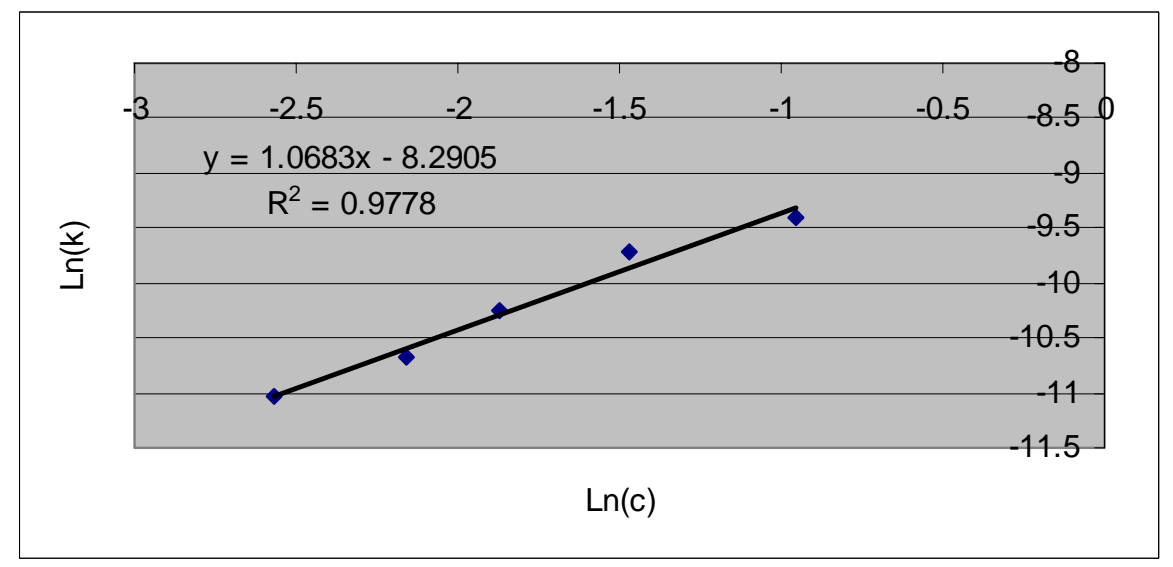

Reaction conditions: ester $=1.0 \mathrm{mmol}$; catalyst $=0.05 \mathrm{mmol}$; total volume $=1.30 \mathrm{~mL}$.

\begin{tabular}{|c|c|c|c|c|}
\hline amine (mol) & amine conc. $\left(\mathrm{mol} \cdot \mathrm{L}^{-1}\right)$ & Initial rate $\mathrm{k}\left(\mathrm{mol} \cdot \mathrm{L}^{-1} \cdot \mathrm{S}^{-1}\right)$ & $\operatorname{Ln}(\mathrm{c})$ & $\operatorname{Ln}(\mathrm{k})$ \\
\hline 0.10 & 0.076923 & 0.0000162 & -2.56495 & -11.0305 \\
\hline 0.15 & 0.115385 & 0.0000229 & -2.15948 & -10.6844 \\
\hline 0.20 & 0.153846 & 0.000035 & -1.8718 & -10.2602 \\
\hline 0.30 & 0.230769 & 0.0000609 & -1.46634 & -9.70628 \\
\hline 0.50 & 0.384615 & 0.0000823 & -0.95551 & -9.40514 \\
\hline
\end{tabular}


Rate order to ester concentration:

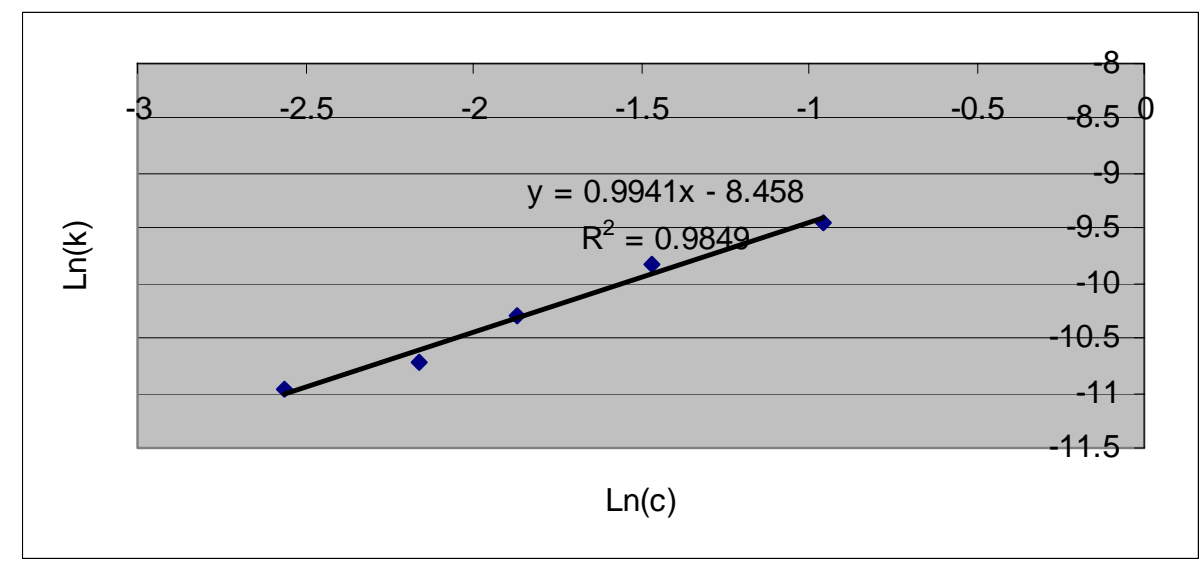

amine $=1.0 \mathrm{mmol}$; catalyst $=0.05 \mathrm{mmol}$; total volume $=1.30 \mathrm{~mL}$.

\begin{tabular}{|c|c|c|c|c|}
\hline ester $(\mathrm{mol})$ & ester conc. $\left(\mathrm{mol} \cdot \mathrm{L}^{-1}\right)$ & Initial rate $\mathrm{k}\left(\mathrm{mol} \cdot \mathrm{L}^{-1} \cdot \mathrm{s}^{-1}\right)$ & $\operatorname{Ln}(\mathrm{c})$ & $\operatorname{Ln}(\mathrm{k})$ \\
\hline 0.10 & 0.076923 & 0.0000174 & -2.56495 & -10.959 \\
\hline 0.15 & 0.115385 & 0.0000222 & -2.15948 & -10.7154 \\
\hline 0.20 & 0.153846 & 0.0000334 & -1.8718 & -10.307 \\
\hline 0.30 & 0.230769 & 0.0000538 & -1.46634 & -9.83024 \\
\hline 0.50 & 0.384615 & 0.0000792 & -0.95551 & -9.44353 \\
\hline
\end{tabular}


Rate order to zirconium concentration:

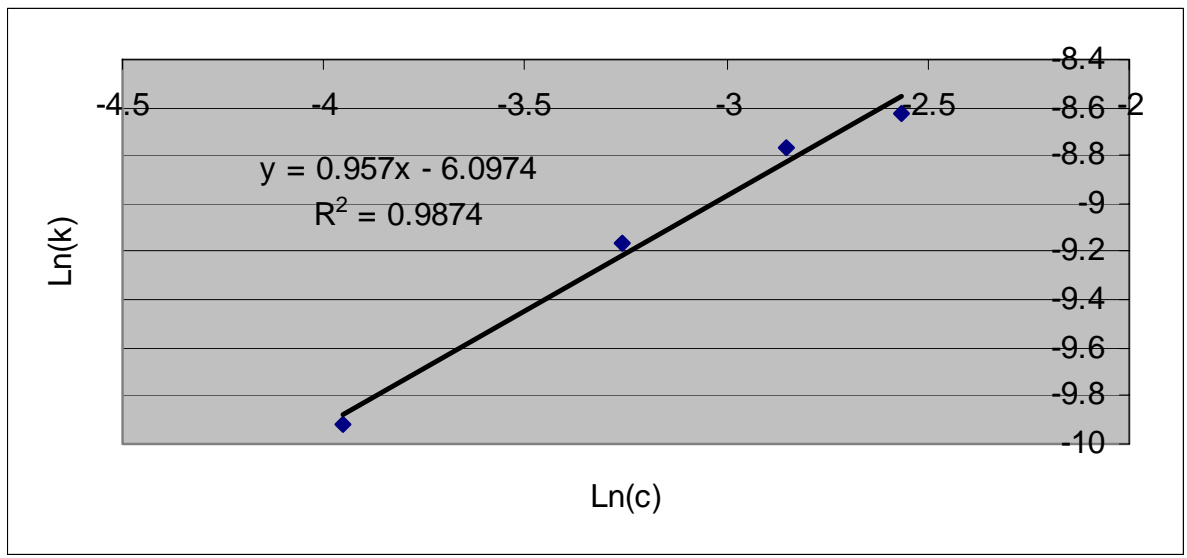

ester $=1.0 \mathrm{mmol} ;$ amine $=1.1 \mathrm{mmol}$; total volume $=1.30 \mathrm{~mL}$.

\begin{tabular}{|c|c|c|c|c|}
\hline cat. (mol\%) & cat. conc. $\left(\mathrm{mol}^{-1}\right)$ & Initial rate $\mathrm{k}\left(\mathrm{mol} \cdot \mathrm{L}^{-1} \cdot \mathrm{s}^{-1}\right)$ & $\operatorname{Ln}(\mathrm{c})$ & $\operatorname{Ln}(\mathrm{k})$ \\
\hline 2.5 & 0.0192308 & 0.0000494 & -3.95124 & -9.91556 \\
\hline 5.0 & 0.0384615 & 0.0000983 & -3.2581 & -9.22749 \\
\hline 7.5 & 0.0576923 & 0.0001558 & -2.85263 & -8.76694 \\
\hline 10.0 & 0.0769231 & 0.0001797 & -2.56495 & -8.62422 \\
\hline
\end{tabular}

